\title{
Space Launch System Booster Separation Aerodynamic Testing in the NASA Langley Unitary Plan Wind Tunnel
}

\author{
Floyd J. Wilcox, Jr.*; Jeremy T. Pinier ${ }^{\dagger}$; and David T. Chan ${ }^{\ddagger}$ \\ NASA Langley Research Center, Hampton, VA 23681 \\ William A. Crosby ${ }^{\S}$ \\ Jacobs ESSSA Group, Huntsville, AL 35812
}

\begin{abstract}
A wind-tunnel investigation of a 0.009 scale model of the Space Launch System (SLS) was conducted in the NASA Langley Unitary Plan Wind Tunnel to characterize the aerodynamics of the core and solid rocket boosters (SRBs) during booster separation. High-pressure air was used to simulate plumes from the booster separation motors (BSMs) located on the nose and aft skirt of the SRBs. Force and moment data were acquired on the core and SRBs. These data were used to corroborate computational fluid dynamics (CFD) calculations that were used in developing a booster separation database. The SRBs could be remotely positioned in the $\mathrm{x}-, \mathrm{y}-$, and $\mathrm{z}$-direction relative to the core. Data were acquired continuously while the SRBs were moved in the axial direction. The primary parameters varied during the test were: core pitch angle; SRB pitch and yaw angles; SRB nose $\mathrm{x}_{-}^{-}, \mathrm{y}_{-}^{-}$, and z-position relative to the core; and BSM plenum pressure. The test was conducted at a free-stream Mach number of 4.25 and a unit Reynolds number of 1.5 million per foot.
\end{abstract}

\section{Nomenclature}

$\begin{array}{ll}\text { alpha1 } & =\text { Core angle of attack, deg } \\ \text { alpha2 } & =\text { RH SRB angle of attack, deg } \\ \text { beta2 } & =\text { RH SRB sideslip, deg } \\ \text { cafc } & =\text { Core axial force coefficient corrected for chamber pressure, body axis } \\ \text { cllfc } & =\text { Core rolling moment coefficient, body axis } \\ \text { clmbr, clmbl } & =\text { RH and LH SRB pitching moment coefficient corrected for thrust tare, body axis } \\ \text { clmfc } & =\text { Core pitching moment coefficient, body axis } \\ \text { clnbr, clnbl } & =\text { RH and LH SRB yawing moment coefficient corrected for thrust tare, body axis } \\ c l n f c & =\text { Core yawing moment coefficient, body axis } \\ c n f b r, c n f b l & =\text { RH and LH SRB normal force coefficient corrected for thrust tare, body axis } \\ c n f c & =\text { Core normal force coefficient, body axis } \\ c y f b r, c y f b l & =\text { RH and LH SRB side force coefficient corrected for thrust tare, body axis } \\ c y f c & =\text { Core side force coefficient, body axis } \\ d p s i b r & =\text { Yaw angle from core body axis to RH SRB body axis (Fig. 16), deg } \\ d t h e t a b r & =\text { Pitch angle from core body axis to RH SRB body axis (Fig. 16), deg } \\ d x b r, d x b l & =\text { Position of RH and LH SRB nose relative to mated position in x-direction (Fig. 14), in. } \\ d y b r, d y b l & =\text { Position of RH and LH SRB nose relative to mated position in y-direction (Fig. 14), in. } \\ d z b r, d z b l & =\text { Position of RH and LH SRB nose relative to mated position in z-direction (Fig. 14), in. }\end{array}$

*Research Aerospace Engineer, Configuration Aerodynamics Branch, MS 499.

${ }^{\dagger}$ Research Aerospace Engineer, Configuration Aerodynamics Branch, MS 499, Associate Fellow AIAA.

${ }^{\ddagger}$ Research Aerospace Engineer, Configuration Aerodynamics Branch, MS 499, Member AIAA.

$\S$ Sub-element Lead for Aerosciencs Support, Aerosciences Branch, Bldg 4600, Member AIAA. 


$\begin{array}{ll}M, \text { Mach } & =\text { Mach number } \\ R e & =\text { Free-stream unit Reynolds number, } \mathrm{ft}^{-1} \\ p_{0} & =\text { Stagnation pressure, psfa } \\ p_{\infty}, \text { pinf } & =\text { Free-stream static pressure, } \mathrm{psfa} \\ q & =\text { Free-stream dynamic pressure, } \mathrm{psfa} \\ \text { pbase1 } & =\text { Core chamber pressure \#1, psfa } \\ \text { pbase2 } & =\text { Core chamber pressure \#2, psfa } \\ \text { pstingbr } & =\text { RH SRB measured sting pressure, psia } \\ T_{0} & =\text { Free-stream stagnation temperature, }{ }^{\circ} \mathrm{F}\end{array}$

Abbreviations and Acronyms

$\begin{array}{ll}\text { AEDC } & =\text { Arnold Engineering Development Complex } \\ \text { AF } & =\text { Axial force, lbf } \\ \text { BLMS } & =\text { Balance Loads Monitoring System } \\ \text { BSM } & =\text { Booster separation motor } \\ \text { CFD } & =\text { Computational fluid dynamics } \\ \text { LAS } & =\text { Launch Abort System } \\ \text { LOX } & =\text { Liquid oxygen } \\ \text { LH } & =\text { Left hand } \\ \text { MRC } & =\text { Moment reference center } \\ \text { NASA } & =\text { National Aeronautics and Space Administration } \\ \text { NF } & =\text { Normal force, lbf } \\ \text { PM } & =\text { Pitching moment, in·lbf } \\ \text { PRT } & =\text { Platinum resistance thermometer } \\ \text { RH } & =\text { Right hand } \\ \text { RM } & =\text { Rolling moment, in·lbf } \\ \text { SF } & =\text { Side force, lbf } \\ \text { SLS } & =\text { Space Launch System } \\ \text { SRB } & =\text { Solid rocket booster } \\ \text { UPWT } & =\text { Unitary Plan Wind Tunnel } \\ \text { YM } & =\text { Yawing moment, in·lbf }\end{array}$

\section{Introduction}

The SLS vehicle consists of a central core and two solid fuel SRBs mounted to each side of the core. After the SRBs complete the boost phase of the launch and have nearly consumed the solid fuel, the SRBs are separated from the core. During the booster separation event, BSMs mounted to the nose and aft skirt of the SRBs fire for approximately 1 second to move the SRBs safely away from the core. The SLS booster separation process is similar to the Space Shuttle booster separation. Additional details of the SLS vehicle and booster separation event can be found in references 1 and 2 .

A wind-tunnel investigation of a 0.009 -scale model of the SLS-10005 configuration was conducted in the Langley Unitary Plan Wind Tunnel to characterize the aerodynamics of the core and SRBs during booster separation. High-pressure air was used to simulate the BSM plumes; however, the core main engine and SRB engine plumes were not simulated. The data obtained from these tests were used to validate CFD calculations of the wind-tunnel scale model 3 [4 4 The CFD codes were then used to compute the aerodynamics of the fullscale vehicle and these results were used to produce the SLS booster separation aerodynamic database $\frac{5] 6}{6}$ The wind-tunnel data were also used in the uncertainty quantification for the database.

During the late 1970s and early 1980s, wind-tunnel tests were conducted at the AEDC Tunnel A to determine the aerodynamic characteristics of the Space Shuttle and SRBs during booster separation ${ }^{7}\left[\begin{array}{l}15 \\ \hline\end{array}\right.$ Some of the hardware used in the Shuttle tests were reused for the current test. The flow-through balances used for the SRBs were refurbished, regauged, and recalibrated for the current test. The SRB separation rig, which was used to position the SRBs relative to the Shuttle, was not available and a new separation rig was fabricated from the original 1970s drawings for use in the current test. 
The wind-tunnel test was conducted at a free-stream Mach number of 4.25 and a free-stream unit Reynolds number of 1.50 million per foot. The test was conducted from August 19, 2014 to October 10, 2014.

\section{Wind-Tunnel Test Description}

\section{A. Wind Tunnel}

The wind-tunnel test was conducted in the Langley UPWT, which is a continuous flow, variable pressure supersonic wind tunnel. The tunnel contains two test sections, which are approximately $4 \mathrm{ft}$ by $4 \mathrm{ft}$ square and $7 \mathrm{ft}$ long. Each test section covers only part of the Mach number range of the tunnel. The nozzle ahead of each test section consists of an asymmetric sliding block, which allows continuous Mach number variation during tunnel operations from 1.5 to 2.9 in the low Mach number test section (\#1) and 2.3 to 4.6 in the high Mach number test section (\#2). The current test was conducted in test section \#2. A complete description of the facility along with test section calibration information can be found in reference 16 .

Table 1 shows the average free-stream condition used during this test in test section \#2. The tunnel air dew point when adjusted to atmospheric pressure was maintained below $-20^{\circ} \mathrm{F}$ to minimize water vapor condensation effects.

Table 1. Approximate tunnel test condition

\begin{tabular}{cccccc}
\hline$M$ & $R e \times 10^{-6}$, per foot & $p_{0}, \mathrm{psfa}$ & $T_{0},{ }^{\circ} \mathrm{F}$ & $q, \mathrm{psfa}$ & $p_{\infty}, \mathrm{psfa}$ \\
\hline 4.25 & 1.5 & 3125 & 150 & 187.5 & 14.8 \\
\hline
\end{tabular}

\section{B. General Installation}

Figures 1 and 2 show the general layout of the core and SRBs mounted in the UPWT. The core and SRBs were installed at a roll angle of $-90^{\circ}$ so that the full capability of the tunnel model support system, which can move longitudinally and laterally, could be used to position the SRBs relative to the core. The core was mounted to the tunnel sidewall using a support strut, which could be manually adjusted to set the core pitch. Because the support strut was located downstream of the test section door, the standard test section door that contains schlieren windows was used so that schlieren images could be acquired during the test. The SRBs were mounted to a separation rig that was attached to the tunnel model support system. The separation rig had the capability to remotely adjust the yaw and y-direction position of the SRBs relative to the core and to manually set the pitch angles of the SRBs. The combination of the separation rig and the tunnel model support system was used to set the attitude and position of the SRBs relative to the core.

High-pressure air was used to simulate the BSM plumes. The high-pressure air was supplied to the SRBs through flexible hoses that connected to the aft end of the SRB stings. The high-pressure air flowed through the stings and into the SRB models. Channels inside the model allowed the air to flow through the SRBs and exit the forward and aft BSMs. A description of the UPWT high-pressure air system will be presented in a subsequent section.

Data were typically acquired in a continuous acquisition mode while moving the SRBs in an axial direction using the model support system. The attitude and position of the SRBs would be set before beginning a run. Table 2 summarizes the core and SRB model position ranges used during the test. The $\mathrm{x}-, \mathrm{y}-$, and z-movements are relative to the SRB nose locations when the SRBs are in the launch (mated) position.

\section{Models and Support Hardware \\ 1. Core}

Figure 3 shows a sketch of the SLS core model detailing the protuberances represented on the model. The model was fabricated in three sections; the aft, middle, and nose. The aft and middle sections were pinned together and were not intended to be separated. The removable nose section allowed access to a model-tobalance sleeve adapter. The majority of the model was fabricated from 17-4 stainless steel. 
Table 2. Summary of core and SRB attitude and position capability

\begin{tabular}{clll}
\hline Model & Capability & Range & Device \\
\hline Core & Pitch & $0^{\circ}, 2^{\circ}$ & Support strut (manually set) \\
SRB & Pitch & $-18^{\circ}$ to $4^{\circ}$ by $2^{\circ}$ & Separation rig (manually set) \\
$\downarrow$ & Yaw (RH SRB) & $-3^{\circ}$ to $10^{\circ}$ & Separation rig (remotely controlled) \\
$\downarrow$ & Yaw (LH SRB) & $3^{\circ}$ to $-10^{\circ}$ & Separation rig (remotely controlled) \\
$\downarrow$ & x-movement & 0 in. to 8 in. & Model support system (remotely controlled) \\
$\downarrow$ & y-movement (RH SRB) & 0 in. to 9.7 in. & Separation rig (remotely controlled) \\
$\downarrow$ & y-movement (LH SRB) & 0 in. to -9.7 in. & Separation rig (remotely controlled) \\
$\downarrow$ & z-movement & -12 in. to 0 in. & Model support system (remotely controlled) \\
\hline
\end{tabular}

\section{SRB Models}

Figure 4 shows a sketch of the right-hand SRB model (left-hand model is a mirror image) with the model protuberances identified. The forward and aft BSM clusters included four nozzles as shown in Fig. 5 . Some runs were conducted with one of the four nozzles in the forward BSM cluster blocked.

The BSM nozzles were designed to match - between flight and wind-tunnel conditions - the nozzle momentum ratio, thrust ratio, initial plume inclination angle, and plume shape $17[18$ The nozzle sizing calculations iterated on the nozzle exit diameter, throat diameter, exit half angle, and chamber pressure. The results of the calculations showed that the nozzle momentum ratio was matched between flight and windtunnel conditions. The total thrust coefficient for the wind-tunnel condition was approximately 1.5 percent larger than the flight condition and the initial plume inclination angle for the wind-tunnel condition was approximately 8.6 percent smaller than the flight condition. Because of the small scale of the wind-tunnel model and the use of high-pressure air as a simulate gas, the shape of the flight and wind-tunnel nozzles were different. Figure 6 compares the flight and wind-tunnel nozzles - drawn at the same scale - to illustrate the different nozzle shapes. The plume boundary (size and shape) for the BSM nozzle at flight and wind-tunnel conditions was computed using the PLUME code 19 The plume boundary at the wind-tunnel condition with a nozzle chamber pressure of 1500 psia matched to within approximately $5 \mathrm{in}$. (full scale) the flight condition plume boundary to $250 \mathrm{in}$. (full scale) downstream of the nozzle.

High-pressure air was used to simulate the BSM plumes. Figure 7 shows a cross section of the RH SRB with the high-pressure air path colored in blue. High-pressure air was fed through a 0.25 in. diameter hole in the center of the sting. At the forward end of the sting, the high-pressure air exited the sting and entered the SRB model through four slots machined perpendicular to the sting bore and oriented $90^{\circ}$ apart. The slots were approximately $0.375 \mathrm{in}$. long and $0.094 \mathrm{in}$. wide.

The high-pressure air then flowed toward the forward and aft BSM clusters. Because the size and length of the forward and aft flow paths through the model were different, the pressure losses through the two flow paths could result in unequal pressures in the forward and aft plenum chambers. Consequently, a flow cap was located in the forward BSM cluster flow path to adjust the flow path area and equalize the pressure in the plenum chambers. Four different flow caps were fabricated to provide different flow areas. A calibration, which used a dedicated BSM cluster insert that contained a pressure orifice to measure the plenum pressure, was conducted at the beginning of the test to determine which flow cap equalized the plenum pressures. The models were designed for a maximum working pressure of 1500 psia and were hydrostatically tested to 2250 psig. The models were primarily fabricated from 17-4 stainless steel.

\section{Boundary Layer Transition Strips}

Grit-type boundary-layer transition strips were applied to the core nose and to each of the SRB noses to ensure fully turbulent boundary layers on the models. The transition strips consisted of \#25 sand grit (0.030 in. dia.) particles single spaced in a line. Two grit bands were applied to the core and one band applied to each of the SRBs. Figure 8 shows the details of the boundary layer grit application. The boundary layer 
grit application duplicated the size and location of grit used during a previous supersonic test of an SLS ascent aerodynamics model.

\section{Separation Rig}

Figure 9a shows the separation rig used to position the SRBs relative to the core. The separation rig was mounted vertically in the test section. Two sting brackets were located on each side of a central gear box. In addition, two Acme screws were located in channels that extended from the gear box to the edge of the rig. Each Acme screw had right-hand threads on one side of the gear box and left-hand threads on the other side. Two electric motors, one for each Acme screw, were mounted under cover plates on either side of the gear box. The sting brackets were attached to the Acme screws with pivot blocks that were threaded onto the Acme screws.

SRB movement was accomplished by rotating the Acme screws, which caused the sting brackets to move either toward or away from the gear box and core in unison. In addition, by varying the rotation speed or direction of one Acme screw relative to the other, the sting brackets could be yawed as shown in Fig. 9b. The sting bracket pivot point was located over the forward Acme screw while the sliding pivot located on the aft screw compensated for the yaw angle. The separation rig provided approximately $10 \mathrm{in}$. of linear travel in the y-direction (see fig. 2 and yaw angles of approximately $-3^{\circ}$ to $10^{\circ}$ (for the RH SRB).

Each SRB was mounted on a balance that was an integral part of a sting. The aft end of each sting was attached to a clutch assembly that was located at the forward part of the sting bracket assembly and was used to set the SRB pitch angles. The clutch assembly consisted of two plates with 180 matching teeth spaced in $2^{\circ}$ increments. The plates were clamped together with a single bolt. The clutch mechanism allowed the booster pitch angles to be set from $-18^{\circ}$ to $2^{\circ}$ in $2^{\circ}$ increments. Figure 10 illustrates an SRB set to a negative pitch angle.

\section{BSM Plume Simulation}

High-pressure air was used to simulate the BSM plumes and was supplied by the UPWT high-pressure air system. Figure 11 shows a schematic of the UPWT high-pressure air system and the basic operating characteristics of the system. High-pressure air is supplied to the UPWT system from a central compressor station and bottle field located on the Langley Research Center. At UPWT, a 5000 psig bottle field is used as a local accumulator and it is constantly resupplied with air from the central compressor station.

The high-pressure air temperature is controlled by directing air through separate hot and cold legs and then recombining the air into a single leg. The hot leg uses a steam heater to heat the air. The air passes through a 5 micron filter before the piping system splits into the two separate legs to prevent debris from contaminating the steam heater coils. Two pneumatic globe valves control the air through the legs in unison - if one valve is set to 75 percent open then the other valve would automatically be set to 25 percent open, i.e., the total percentage valve opening is always 100 percent when air is flowing. The air from the two legs are combined before passing through a second 5 micron filter.

Pressure in the system is controlled with a digital control valve. This valve contains 16 on/off valves with the flow passage area of each valve doubling from from smallest to the largest valve. After passing through the digital control valve, the air is then piped to manifolds located in the east and west balance box on either side of test section \#2. The balance boxes are chambers that house the model support system gears and shafts used to move the system laterally and longitudinally.

Figure 12 shows a schematic diagram of the high-pressure air routing from the UPWT high-pressure air system manifold in the balance box to the SRB models. Because of the significant amount of piping from the steam heater to the SRB models, it was necessary to preheat the piping before beginning a run so that the temperature of the flow would not decrease before reaching the SRBs. The mass flow required through the SRBs was relatively small compared to the mass flow that can flow through the primary piping of the highpressure air system so preheating the system through the SRBs would have taken a significantly long period of time. Therefore, a remotely actuated by-pass valve was located on the manifold with piping exhausting the air into the tunnel flow downstream of the model support system. When this valve was opened, the mass flow passing through the high-pressure air system heated the piping to the proper temperature in approximately 5 minutes. In addition, when the by-pass valve was open, some air was also passing through

the piping from the manifold to the SRBs and conditioning that part of the system. During BSM plume simulation runs, the stagnation temperature of the high-pressure air was approximately $150^{\circ} \mathrm{F}$.

5 of 31

American Institute of Aeronautics and Astronautics 
The high-pressure air from the manifold to the SRBs passed through a non-critical venturi flow meter to measure the total mass flow through both SRBs. The venturi was instrumented with a $200 \mathrm{psid} \Delta \mathrm{p}$ transducer and a 2000 psia static pressure transducer. The temperature of the air flowing through the venturi was measured with a type $\mathrm{T}$ (copper-constantan) thermocouple located in the manifold. After passing through the venturi, the air was split at a tee fitting for the left- and right-hand SRBs. Two flexible stainless steel braided hoses were connected to the tee fitting and the SRB stings. The hoses were approximately $15.5 \mathrm{ft}$ long and had an inside diameter of $0.402 \mathrm{in}$. The flexible hoses were used to allow movement of the SRBs during the test.

The pressure inside each sting was measured with a 2000 psia pressure transducer at a location near the end of each sting. The sting pressures were used to set the plenum pressures for the BSM clusters based on the results obtained during the flow cap calibration runs discussed previously. BSM plume simulation runs were conducted at four pressures which correspond to full scale BSM cluster thrust levels of nominal, high, low, and ramp down.

\section{Instrumentation and Measurements}

\section{Force and Moment Balances}

CORE MODEL. Force and moment data on the core model were measured with a 6-component electrical strain gauge balance (Langley NTF-119). Table 3 shows the full scale balance loads. Three 4-wire PRT's were attached to the balance to monitor the balance temperature and the balance thermal gradients. The measured balance temperatures were not used to adjust the balance sensitivities.

Table 3. Load range of core and SRB balances

\begin{tabular}{lll}
\hline Balance component & Core load range & SRB load range \\
\hline normal force & $\pm 300 \mathrm{lbf}$ & $\pm 125 \mathrm{lbf}$ \\
axial force & $\pm 120 \mathrm{lbf}$ & $\mathrm{N} / \mathrm{A}$ \\
pitching moment & $\pm 810 \mathrm{in} \cdot \mathrm{lbf}$ & $\pm 450 \mathrm{in} \cdot \mathrm{lbf}$ \\
rolling moment & $\pm 210 \mathrm{in} \cdot \mathrm{lbf}$ & $\mathrm{N} / \mathrm{A}$ \\
yawing moment & $\pm 810 \mathrm{in} \cdot \mathrm{lbf}$ & $\pm 450 \mathrm{in} \cdot \mathrm{lbf}$ \\
side force & $\pm 300 \mathrm{lbf}$ & $\pm 125 \mathrm{lbf}$ \\
\hline
\end{tabular}

A Balance Loads Monitoring System (BLMS), which displays the balance loads for each component in real time, was connected to the NTF-119 balance throughout the entire test. The BLMS consists of a personal computer running software that computes the balance forces and moments through interactions and displays the results in real time so that the balance loading dynamics can be monitored. In addition, the BLMS will sound an alarm when the balance loads exceed 80 percent of the balance load limits. During this test, several incidents of severe core model fluctuations occurred when the SRBs were in proximity to the core and high-pressure air was blowing through the BSMs. Further discussion of the core model dynamics is presented in the Results section.

SRB MODELS. Force and moment data on each SRB model were measured with a 4-component (excluding axial force and rolling moment) electrical strain gauge sting/balance. Each balance was designed to be a flow through balance to allow high-pressure air to pass through the sting/balance to the SRB models. These balances were the property of the AEDC and on loan to NASA for this test.

Table 3 shows the full scale loads for the SRB balances. Two 4-wire PRT's were attached to each balance to monitor the balance temperature and the balance thermal gradients. The measured balance temperatures were not used to adjust the balance sensitivities. The AEDC balances were re-gaged and calibrated prior to the test. The balances were designed for a maximum working pressure of 1500 psia and were hydrostatically tested to 2250 psig. 


\section{Core Chamber Pressure}

The core chamber pressure was measured with two pressure tubes located on either side of the sting and extending approximately 4 inches inside the base of the core. Figure 13 shows the location of two chamber pressure tubes. Each chamber pressure was measured with a 5 psia pressure transducer. Both chamber pressure readings were averaged and applied to a single chamber area. The core base was modified with an inside chamfer to eliminate the model base area.

\section{Delta SRB Nose and Angular Positions}

The LH and RH SRB delta nose positions are computed relative to axis systems that are aligned with the core body axis system and are located at the nose of the SRBs when the SRBs are in the launch (mated) configuration. Figure 14 shows the orientation and location of the delta nose position axis systems. Figure 15 shows a three-dimensional view of the RH SRB delta positions.

The delta nose positions were computed by calculating the nose positions of the core and SRBs relative to an arbitrary axis system aligned with the tunnel. These nose positions were then converted to an axis system aligned with the core body axis system. The SRB nose positions in the launch position were then computed from the launch configuration dimensions. Finally, the delta SRB nose positions were computed by subtracting the SRB nose positions in the launch position from the separated SRB nose positions.

The SRB delta angular positions are the yaw, pitch, and roll rotations from the core body axis system to the RH and LH SRB body axis systems. Figure 16 shows the yaw (psicrh) and pitch (thetacrh) rotations for the right-hand SRB from the core. The figure shows a positive yaw and negative pitch. The angular positions for the LH SRB are similarly defined.

\section{Data Acquisition}

Most of the SRB separation data were acquired in a continuous data acquisition mode of operation. In this mode, data are continuously acquired while the SRB models were continuously moved in the tunnel downstream direction. Although most of the separation data were acquired in a continuous mode, some data were acquired in a move-pause mode of operation. The general procedure used to acquire continuous data is described below:

1. Position the right-hand SRB at the proper position and attitude relative to the core with $d x b r=0$ in.

2. Begin continuous data acquisition.

3. Begin continuous axial movement of SRBs.

4. Stop data acquisition system after axial movement of SRBs is completed.

For the continuous data acquisition mode, data were scanned at a rate of 30 frames per second. A typical run covered approximately 8 in. of downstream movement and required approximately 40 seconds to complete.

For the move-pause data acquisition mode, data were scanned at a rate of 30 frames per second for two seconds. The 60 frames of data were averaged before data reduction.

\section{Corrections}

The tunnel flow angularity was assumed to be zero for this test. The core force and moment data have been corrected to the condition of free-stream static pressure acting over the core chamber area. The angular position and nose position of the core and SRBs have been corrected for sting and balance deflections caused by aerodynamic loads.

The forces and moments on the SRBs have been corrected for BSM thrust tares by subtracting the thrust tare from the measured wind-on forces and moments. The thrust tares were determined from calibrations performed during wind-off conditions with the tunnel pressure held constant at $290 \mathrm{psfa}$, which was the lowest pressure that the tunnel vacuum pumps could maintain while high-pressure air was flowing through the BSMs and into the tunnel. 


\section{E. Run Matrix}

A total of 585 wind-on runs were conducted during this test. Because most of the data were acquired in a continuous data acquisition mode, more than 12 million data points were acquired. The total size of the data files was greater than 7.9 GB.

The primary parameters varied during the test are shown below.

- Model attitudes

- Core pitch $\Longrightarrow 0^{\circ}, 2^{\circ}$

- SRB pitch $\Longrightarrow-18^{\circ}$ to $4^{\circ}$

$-\mathrm{RH}$ SRB yaw $\Longrightarrow-3^{\circ}$ to $10^{\circ}$

- SRB model positions (model scale)

$-d x b r \Longrightarrow 0$ in. to 8 in.

$-d y b r \Longrightarrow 0$ in. to 10 in.

$-d z b r \Longrightarrow-12$ in. to 0 in.
- SRB model attitude relative to core

$$
- \text { dpsibr } \Longrightarrow-3^{\circ} \text { to } 10^{\circ}
$$$$
- \text { dthetabr } \Longrightarrow-18^{\circ} \text { to } 1^{\circ}
$$

Figure 17 shows all of the RH SRB $d y b r$ and $d z b r$ nose position combinations that were tested. Many of the points have multiple symbols at the same location, which indicate runs with the SRBs at different attitudes but with noses at the same position. When the SRBs were close to the core, the nose positions were clustered close together; however, when the SRBs were further from the core, the tested nose positions were spread apart to account for the extent of possible SRB trajectory bounds.

Figure 18 shows all of the RH SRB dpsibr and dthetabr attitude combinations that were tested. Again, many of the points have multiple symbols at the same location, which indicate runs with the SRBs at different nose positions but at the same attitude. When the SRBs were close to the core, the tested SRB attitudes were relatively small, whereas when the SRBs were further from the core, the range of attitudes was larger.

\section{Results}

\section{A. Core Model Dynamics}

Significant core model dynamics occasionally occurred during the test and caused the core model to primarily shake in the core pitch plane (horizontal plane). In some circumstances, the core model balance load limits were exceeded for some components. The model dynamics only occurred during BSM plume simulation runs and when the SRBs were near the core. When preparing for BSM plume simulation runs, the RH SRB $d x b r$ was set to zero and $d z b r$ was set to the proper value for the upcoming run. The RH SRB $d y b r$ was set to a large value and then decreased to move the SRB toward the core. The core model dynamics typically occurred as the SRBs were moved toward the core in the dybr direction. When model dynamics did occur, that particular run was either skipped or the SRBs were set further away from the core to prevent the dynamics.

A few continuous sweep runs were started and then aborted when the core model dynamics began as $d x b r$ increased. To stop the model dynamics quickly, the separation rig forward and aft screws were operated in opposite directions to cause the SRB noses to quickly move away from the core.

It is unclear if the core model dynamics were simply a result of unsteady pressures on the core that excited the flexible core model/balance/sting system or if it was a significant dynamic event that might occur on the actual vehicle. The dynamic event, which would occur over approximately 1 second (duration of the full-scale vehicle BSMs firing), would have to overcome the significant inertia of the full-scale SLS vehicle at booster separation.

\section{B. Core Nose Shock Location}

During BSM plume simulation runs, a strong core nose shock (a shock that is more normal - less sweptthan the shocks on the core with no BSM plume simulation) would be either fixed to the LAS, capsule, or jumping between the LAS and capsule. In some cases, the core nose shock would abruptly jump from the LAS to the capsule. 
Figure 19 shows the core aerodynamic coefficients as a function of $d x b r$ for a continuous run and a repeat move-pause run with BSM plume simulation. The axial force coefficient shows a sharp increase between $d x b r$ of 6 in. and $7 \mathrm{in}$. Pitching and yawing moment coefficients show a small discontinuity at the same $d x b r$ position. The remaining coefficients do not show any effect. The axial force coefficient jump was caused by the core nose shock abruptly jumping from the LAS tip to the capsule. Figure 20 shows schlieren images for the move-pause run at $d x b r$ of 6 in. and $7 \mathrm{in}$., in addition, the figure shows the core alone schlieren for comparison.

The core nose shock did not always jump abruptly as shown in Fig. 19. In some cases, the core nose shock jumped from the LAS tip to the capsule and back to the LAS tip repeatedly. Figure 21 shows the core aerodynamic coefficients as a function of $d x b r$ for a case where the core nose shock was jumping between the LAS tip and capsule for 0 in. $\leq d x b r \lesssim 2.2$ in. The longitudinal aerodynamic coefficients show the effect of the core nose shock jumping. The lateral aerodynamic coefficients show either no effect or a small effect. Because the nose shock was rapidly jumping between the LAS tip and capsule, it is possible that the shock movement was not symmetrical, which caused the effect to be seen in not only the axial force coefficient but also the normal force and pitching moment coefficients.

Because the location of the core nose shock can affect the aerodynamic characteristics of the core and the SRBs, knowing where the core nose shock is located and whether it is fixed or jumping is important for creating a separation database. Figure 22 shows a series of plots that indicate the location of the core nose shock for all of the BSM plume simulation runs (with all BSM nozzles open). Each of the 15 small plots shows the RH SRB nose position in terms of $d y b r$ and $d z b r$ at $d x b r$ values from 0 in. to $3.50 \mathrm{in}$. in 0.25 in. increments. The core nose shock location is indicated by the three color coded symbols. The plotted points include all of the core and SRB pitch and yaw attitude variations. Using the figure, the change in the core nose shock location can be discerned as the RH SRB moves aft (increasing $d x b r$ ). For example, at $d x b r=0$ in. and $d y b r=0.5$ in., there are a number of cases with -2.5 in. $\gtrsim d z b r \gtrsim-5$ in. where the core nose shock is classified as jumping (green squares). As $d x b r$ increases to 3.25 in., the core nose shock is now located at the capsule for all of these cases (blue diamonds).

Figure 22 also shows an orange box on each $d x b r$ plot except at $d x b r=0$ in. The box indicates the separation trajectory bounds that were computed from a Guidance, Navigation, and Control (GN\&C) simulation of the booster separation using an existing preliminary separation database. The center of the box represents the nominal SRB separation trajectory. A solid orange box indicates that the BSMs are firing and a dashed box indicates that the BSMs are not firing. On the actual vehicle, the BSMs fire for approximately 1 second; therefore, at model scale, the BSMs would only fire while $d x b r \lesssim 1 \mathrm{in}$. From $d x b r=0$ in. to 1 in., the GN\&C separation trajectory bounds only include cases where the core nose shock is located on the capsule, although there are some cases near the box edges that are near cases where the core nose shock is on the LAS. These results suggest that the core nose shock location would generally be located on the capsule for the expected SRB separation trajectory bounds. Consequently, the generation of the separation database can be simplified by having to only consider one core nose shock location. However, after creating a separation database, it would be prudent to compute the separation trajectory bounds with the new database and plot the new bounds with the experimental data to ensure that the bounds only include cases where the core nose shock is on the capsule.

Although the position of the SRB nose relative to the core is a significant indicator of whether the core nose shock is fixed to the LAS tip or capsule, the BSM plenum pressure also affected the position of the core nose shock. A series of schlieren photographs were acquired with the SRBs at a fixed position and attitude while the BSM plenum pressure was increased in steps of 100 psia. The SRB position and attitude are shown below.

- $d x b r=0$ in.

- $d y b r=0.5$ in.

- $d z b r=-0.5$ in.

- $d p s i b r=0.1^{\circ}$

- dthetabr $=-1.4^{\circ}$

Figure 23 shows two schlieren images with the core nose shock jumping from the capsule to the LAS tip when the BSM plenum pressure was increased from approximately 1100 psia to 1200 psia. 


\section{Repeatability}

Multiple single repeat runs were acquired throughout the test and were used in the separation database uncertainty quantification. In addition to these single repeat runs, four sets of three back-to-back repeat runs - for configurations with and without BSM plume simulation - were acquired during the test to provide an indication of data repeatability throughout the test. Each set of repeat runs was obtained after numerous model changes had occurred. Only the repeat runs with BSM plume simulation are presented in this paper.

Figure 24 shows the first set of back-to-back repeat runs for the core and SRBs with BSM plume simulation. The error bars in the plots are the balance calibration accuracies converted to coefficient form and provide an order-of-magnitude comparison of the repeat run scatter. The error bars on the core pitching and yawing moments are smaller than the symbols and are not shown. The repeatability shown in Fig. 24 was typical for the remaining sets of repeat runs and indicates that there were no significant repeatability issues during the test. However, during the third and forth sets of back-to-back repeat runs, the first run in the set showed a significant shift in core axial force because the core nose shock jumped from the LAS tip to the capsule. Figure 25 shows the third set of back-to-back repeats. The core nose shock jump from the LAS tip to the capsule is also evident in the core normal force, pitching moment, and in both the RH and LH SRB pitching and yawing moments. The core nose shock jump from the LAS tip to the capsule during the first run of the third and fourth sets of repeat runs indicate that the core nose shock position is not only dependent on the SRB nose position, as discussed in the previous section, but also on the path used to position the SRB for the first run in the repeats.

\section{Blockage}

When the BSM plumes were not simulated, the core chamber pressures were sometimes lower than freestream static pressure as expected but during other runs the chamber pressures were higher than free-stream static pressure. Figure 26 shows the core chamber pressures for runs without BSM plume simulation where the chamber pressures were below and above free-stream static pressure. The two cases were nearly identical except that the run with chamber pressures above free-stream static pressure were obtained with a $d z b r$ closer to the core. When the BSM plumes were simulated, the core chamber pressures were generally higher

than free-stream static pressure. Figure 27 shows the core chamber pressures for runs with BSM plume simulation for the same cases as Fig. 26 except dthetabr is more negative because of pitch deflection caused by the BSM thrust.

The results shown in Fig. 26 (without BSM plume simulation) indicate that flow blockage is occurring between the separation rig and core support strut and high-pressure air is feeding upstream to the base of the core as evidenced by the increase in core chamber pressures. For the cases with BSM plume simulation, the high-pressure air from the aft BSM cluster is causing the increase in core chamber pressures.

Figure 28 shows all of the runs with core chamber pressures above and below free-stream static pressure for the cases without BSM plume simulation as a function of $d y b r$ and $d z b r$. The core chamber pressure in Fig. 28 was the average pressure over the entire run. The results show that the core chamber pressure was generally above free-stream static pressure for the runs where the SRBs were closer to the core $(d z b r \lesssim 4$ in.) although there are some runs at larger $d z b r$ and $d y b r$ values.

Figure 29 shows the SRB separation rig positioned close to the core support strut illustrating the lack of a flow path between the separation rig and core support strut. Because of the flow blockage issue, the runs without BSM plume simulation and with core chamber pressures above free-stream static pressure were not used in the SLS booster separation database uncertainty quantification calculations.

Booster separation tests for the Shuttle probably did not have issues with flow blockage because of the configuration of the SRBs and external tank. Figure 30 shows a sketch from a Shuttle booster separation test that was conducted in the 1970s $!^{7}$ The Shuttle was attached to an offset sting and the external tank was attached to the Shuttle. Consequently, when the SRBs were in the mated position along side the external tank, the separation rig was approximately 3.7 in. below the Shuttle sting allowing a significant flow path between the separation rig and the Shuttle support system.

\section{Concluding Remarks}

A wind-tunnel investigation of a 0.009-scale SLS-10005 model was conducted in the Langley UPWT to characterize the aerodynamics of the core and SRBs during booster separation at a Mach number of 
4.25. The SRB BSM plumes were simulated with high-pressure air; however, the core main engine plumes and SRB engine plumes were not simulated. These data were used to validate CFD calculations of the wind-tunnel scale model before using the CFD codes to compute the aerodynamics of the full-scale vehicle for use in developing an SLS booster separation aerodynamic database. The wind-tunnel data were also used in the database uncertainty quantification. The primary parameters that were varied during the test include: core pitch angle; SRB pitch and yaw angles; SRB nose $\mathrm{x}^{-}, \mathrm{y}^{-}$, and $\mathrm{z}-$-position relative to the core; and BSM plenum pressure. A total of 585 wind-on runs were completed. A summary of important findings are provided below.

1. Significant core model dynamics occasionally occurred during BSM plume simulation runs when the SRBs were close to the core. It is unclear whether the dynamics would be an issue for the full scale vehicle.

2. The core nose shock position was dependent on the position and attitude of the SRBs relative to the core and on the BSM plenum pressure. In addition, the path used to position the SRBs at the beginning of a run sometimes affected the core nose shock position.

3. Multiple back-to-back repeat runs conducted throughout the test indicated no significant repeatability issues.

4. Blockage between the core support strut and separation rig probably occurred for some runs as evidenced by the increase in core chamber pressures. The blockage typically occurred when the SRBs were close to the launch (mated) position.

\section{References}

\footnotetext{
${ }^{1}$ Blevins, John A.; Campbell, John R., Jr.; Bennett, David W.; Raush, Russ D.; Gomez, Reynaldo J.; and Kiris, Cetin C.: An Overview of the Characterization of the Space Launch System Aerodynamic Environments. AIAA 2014-1253, 2014.

${ }^{2}$ Pritchett, Victor E.; Mayle, Melody N.; Blevins, John A.; Crosby, William A.; and Purinton, David C.: Aerodynamic Tests of the Space Launch System Database Development. AIAA 2014-1256, 2014.

${ }^{3}$ Rogers, Stuart E.; Dalle, Derek J.; and Chan, William M.: CFD Simulations of the Space Launch System Ascent Aerodynamics and Booster Separation. AIAA 2015-0778, 2015.

${ }^{4}$ Dalle, Derek J.; and Rogers, Stuart E.: Output-Based Adaptive Meshing Applied to Space Launch System Booster Separation Analysis. AIAA 2015-3152, 2015.

${ }^{5}$ Dalle, Derek J.; Rogers, Stuart E.; Chan, William M.; and Lee, H.: Inviscid and Viscous CFD Analysis of Booster Separation for the Space Launch System Vehicle. 54th AIAA Aerospace Sciences Meeting, Jan. 2016.

${ }^{6}$ Chan, David T.; Dalle, Derek J.; Pinier, Jeremy T.; Rogers, Stuart E.; Wilcox, Floyd J., Jr.; and Gomez, Reynaldo J.: Development and Uncertainty Quantification of the Space Launch System Near-Proximity Booster Separation Aerodynamic Database. 54th AIAA Aerospace Sciences Meeting, Jan. 2016.

${ }^{7}$ Daileda, J. J.; and Marroquin, J.: Results of Tests Using a 0.010-Scale SSV Model 75-OTS in the AEDC VKF Tunnel A (IA40). NASA CR-151381 (DMS-DR-2293), 1977.

${ }^{8}$ Daileda, J. J.; and Marroquin, J.: Results of SRB Separation Tests Using the 0.010-Scale SSV Model 75-OTS in the AEDC VKF Tunnel A (IA142); Volume 1 of 3. NASA CR-151385 (DMS-DR-2346), 1977.

${ }^{9}$ Daileda, J. J.; and Marroquin, J.: Results of SRB Separation Tests Using the 0.010-Scale SSV Model 75-OTS in the AEDC VKF Tunnel A (IA142); Volume 2 of 3. NASA CR-151386 (DMS-DR-2346), 1977.

${ }^{10}$ Daileda, J. J.; and Marroquin, J.: Results of SRB Separation Tests Using the 0.010-Scale SSV Model 75-OTS in the AEDC VKF Tunnel A (IA142); Volume 3 of 3. NASA CR-151387 (DMS-DR-2346), 1977.

${ }^{11}$ Daileda, J. J.; and Marroquin, J.: Results of SRB Separation Tests Using the 0.010-Scale SSV Model 75-OTS in the AEDC VKF Tunnel A (IA143); Volume 1 of 4. NASA CR-151401 (DMS-DR-2354), 1978.

${ }^{12}$ Daileda, J. J.; and Marroquin, J.: Results of SRB Separation Tests Using the 0.010-Scale SSV Model 75-OTS in the AEDC VKF Tunnel A (IA143); Volume 2 of 4. NASA CR-151402 (DMS-DR-2354), 1978.

${ }^{13}$ Daileda, J. J.; and Marroquin, J.: Results of SRB Separation Tests Using the 0.010-Scale SSV Model 75-OTS in the AEDC VKF Tunnel A (IA143); Volume 3 of 4. NASA CR-151403 (DMS-DR-2354), 1978.

${ }^{14}$ Daileda, J. J.; and Marroquin, J.: Results of SRB Separation Tests Using the 0.010-Scale SSV Model 75-OTS in the AEDC VKF Tunnel A (IA143); Volume 4 of 4. NASA CR-151404 (DMS-DR-2354), 1978.

${ }^{15}$ Crosby, W. A.; and Lanham, D. L.: Integrated Aerodynamic Tests of the Space Shuttle Vehicle During Solid Rocket Booster Separation at Mach 4.5 (IA193). AEDC-TSR-82-V15, 1982.

${ }^{16}$ Jackson, Charlie M., Jr.; Corlett, William A.; and Monta, William J.: Description and Calibration of the Langley Unitary Plan Wind Tunnel. NASA TP-1905, 1981.

${ }^{17}$ Brauckmann, Gregory J.; Greathouse, James S.; and White, Molly E.: Rocket Plume Scaling for Orion Wind Tunnel Testing. AIAA 2011-3341, 2011.

${ }^{18}$ Pindzola, M.: Jet Simulation in Ground Test Facilities. AGARDograph79, 1963.

${ }^{19}$ Salas, Manuel D.: The Numerical Calculations of Inviscid Plume Flow Fields. AIAA 1974-0523, 1974.
} 


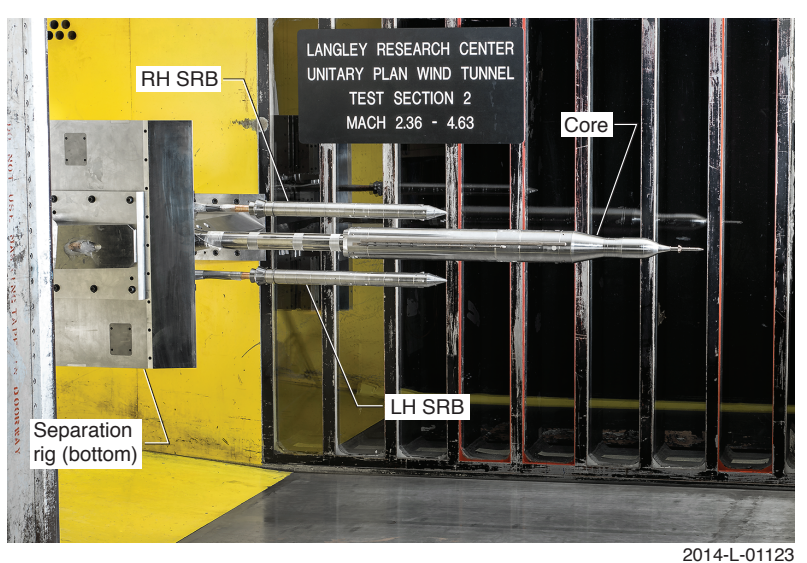

(a) Side view.

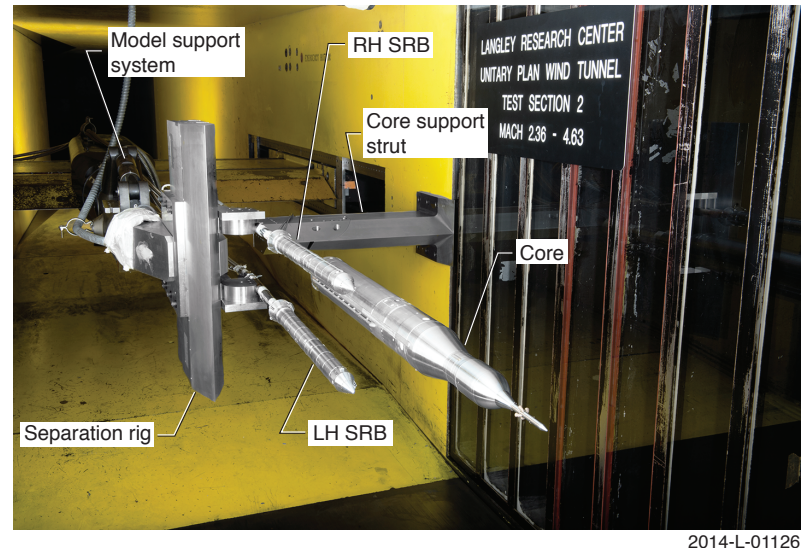

(b) View looking downstream.

Figure 1. Installation photographs showing the general model setup.

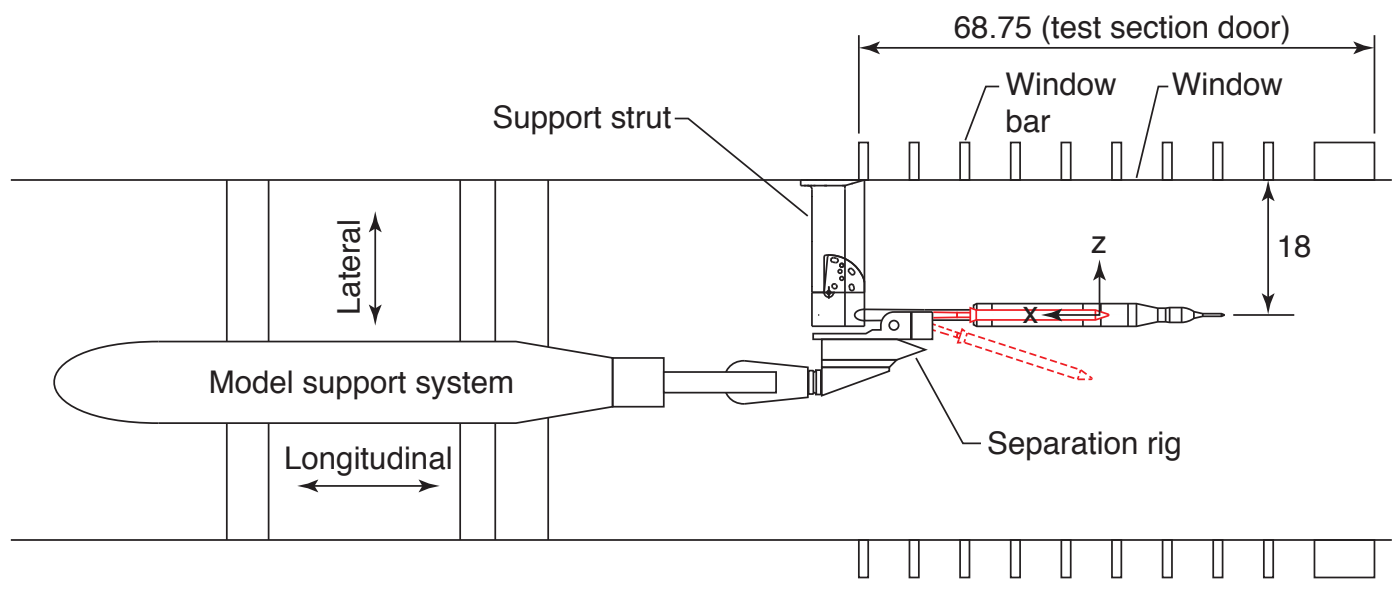

(a) Top view.

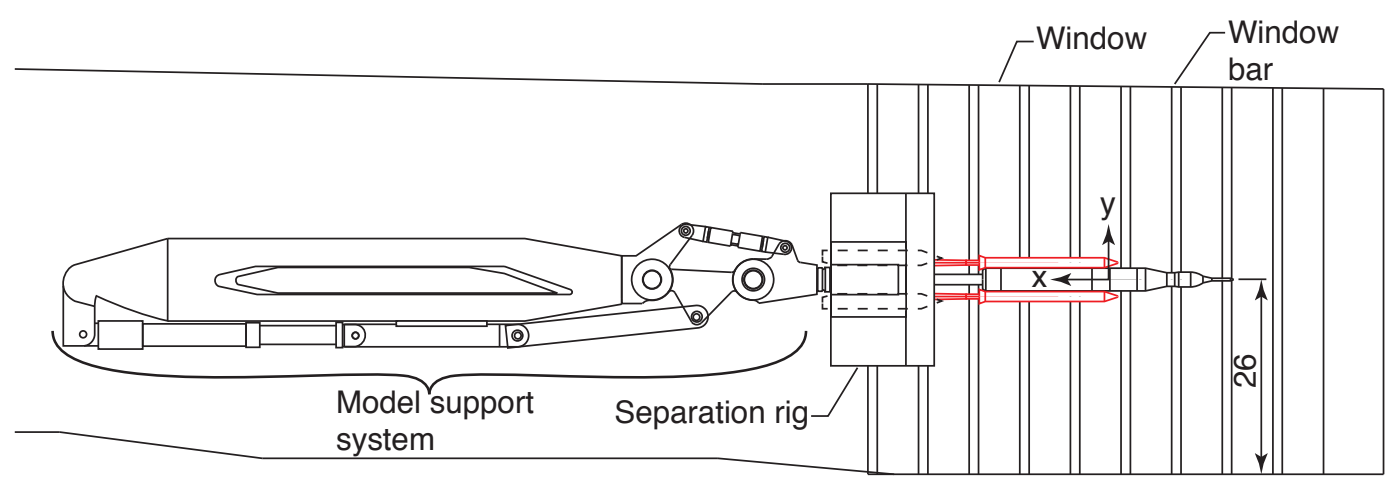

(b) Side view.

Figure 2. Installation sketches showing the general model setup. All dimensions are in inches. 


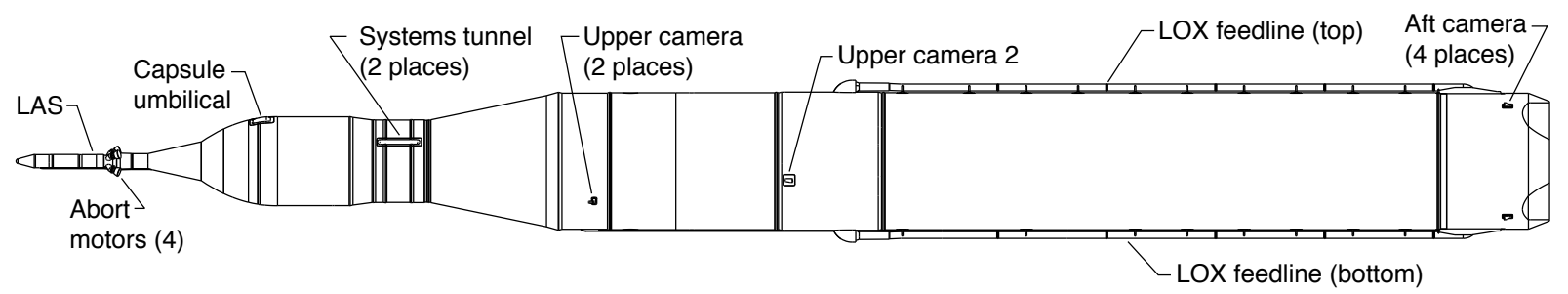

(a) Left-hand side view.

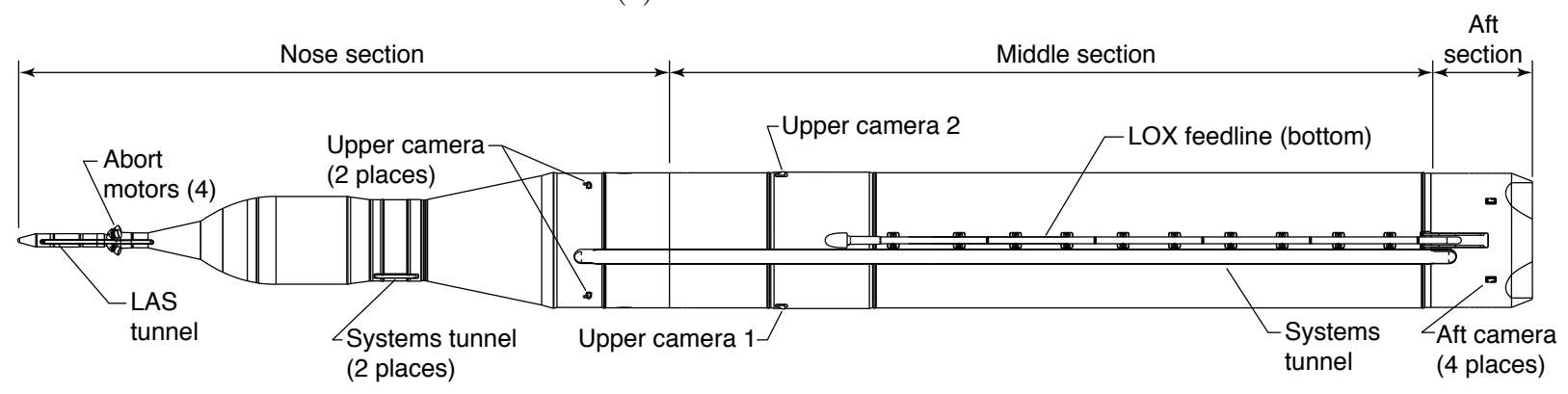

(b) Bottom view.

Figure 3. SLS core model.

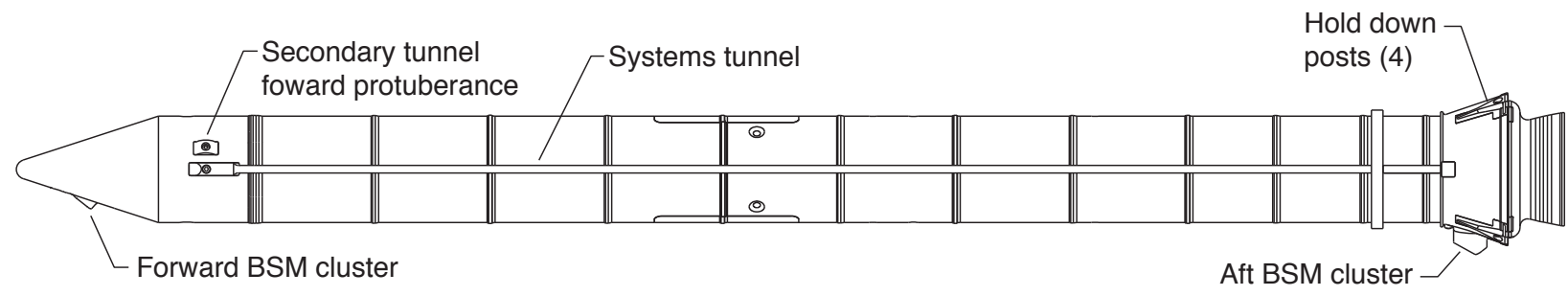

(a) Right-hand side view.

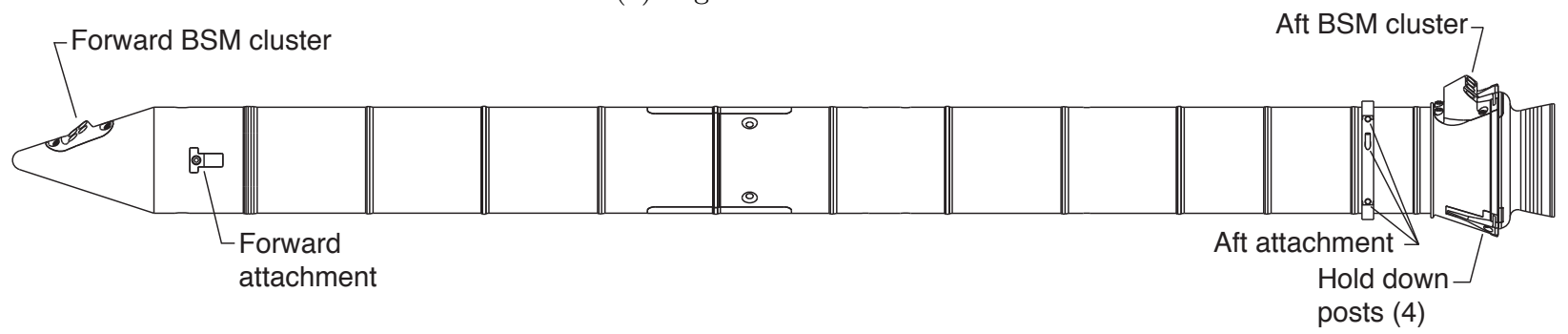

(b) Left-hand side view.

Figure 4. Right-hand SRB model (left-hand SRB similar). 


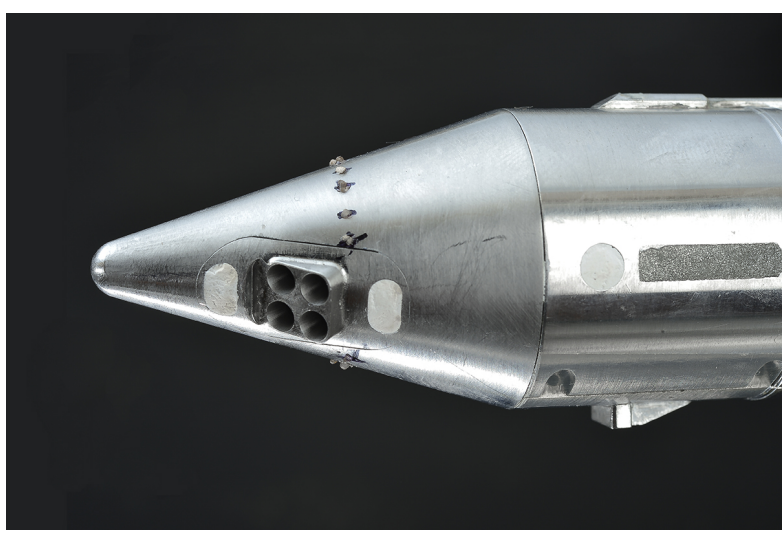

(a) Forward BSM cluster.

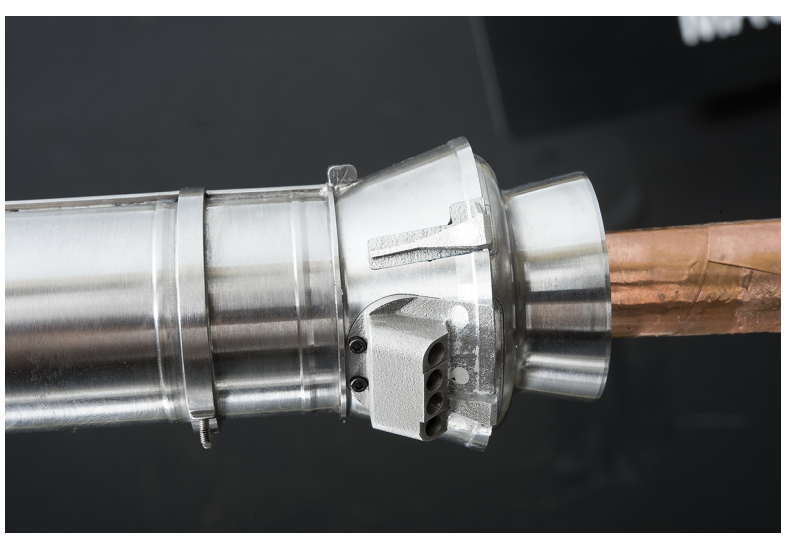

(b) Aft BSM cluster.

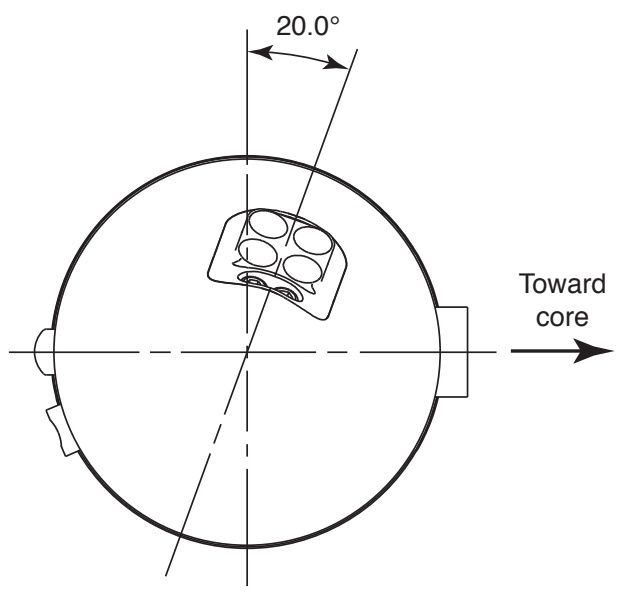

(c) Orientation of forward BSM cluster (looking downstream). Aft BSM cluster similar.

Figure 5. Right-hand SRB model BSM clusters.

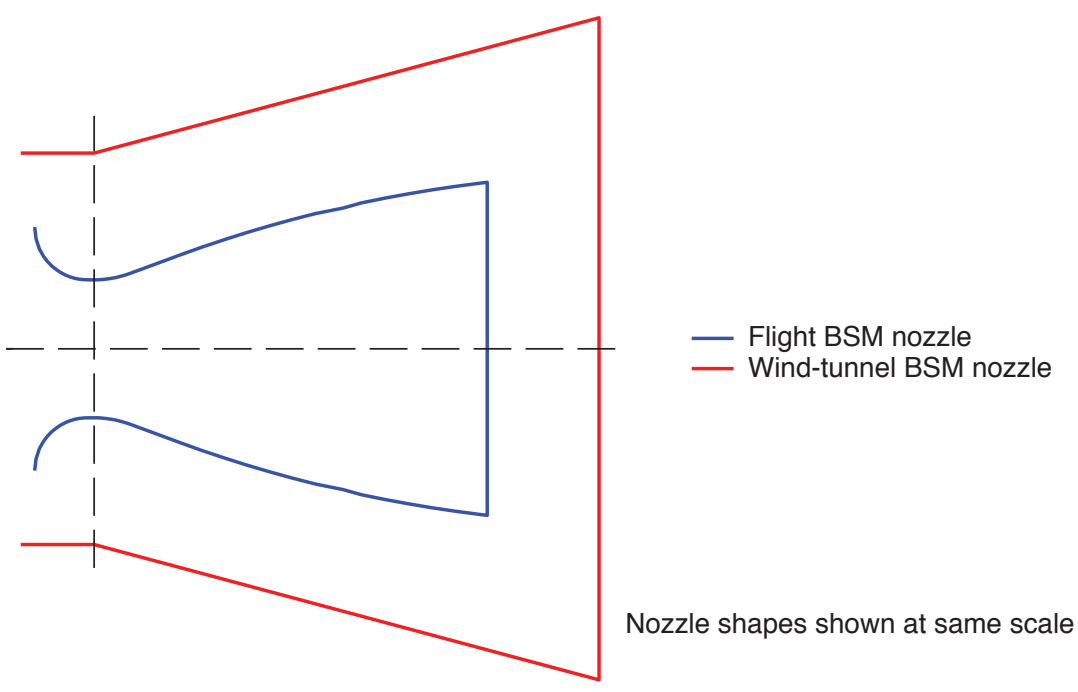

Figure 6. Comparison of flight and wind-tunnel BSM nozzle shapes. 


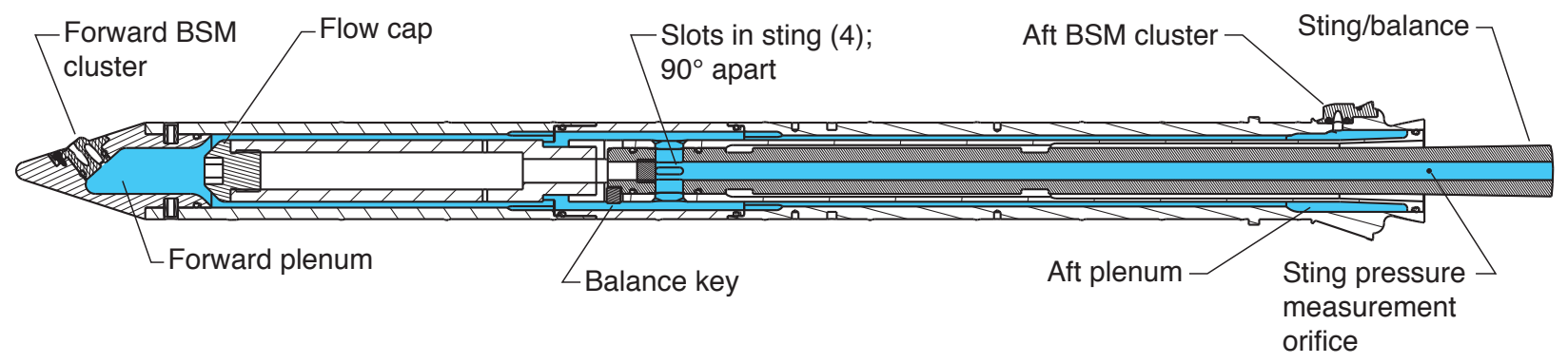

Figure 7. Right-hand SRB model cross section illustrating high-pressure air path.

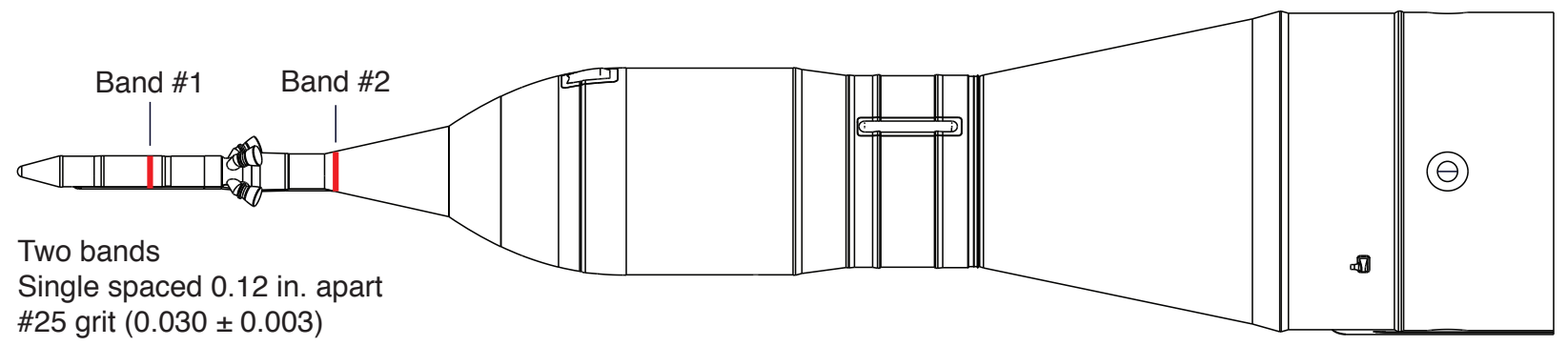

(a) SLS core.

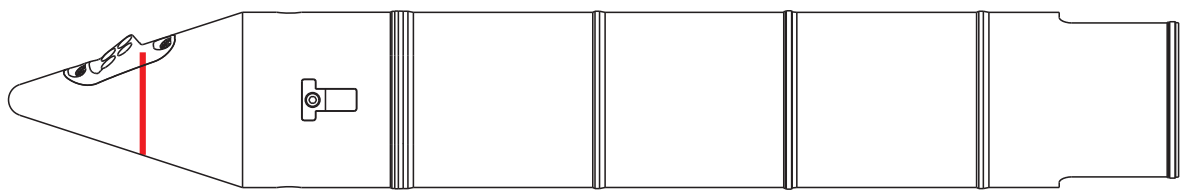

Single spaced 0.12 in. apart $\# 25$ grit $(0.030 \pm 0.003)$

(b) RH SRB (LH SRB similar).

Figure 8. Boundary layer transition grit size and location. 


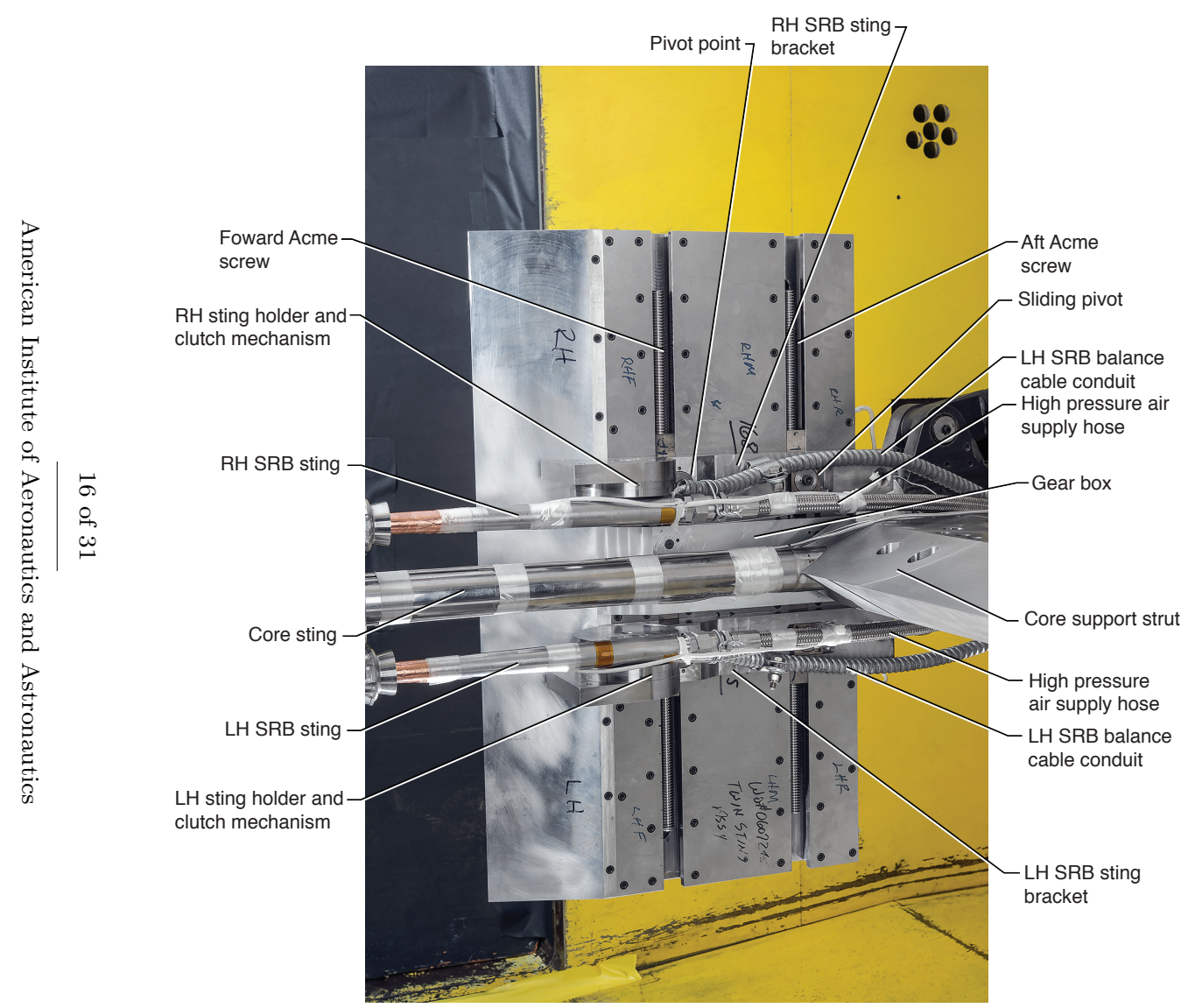

(a) Parts identification.

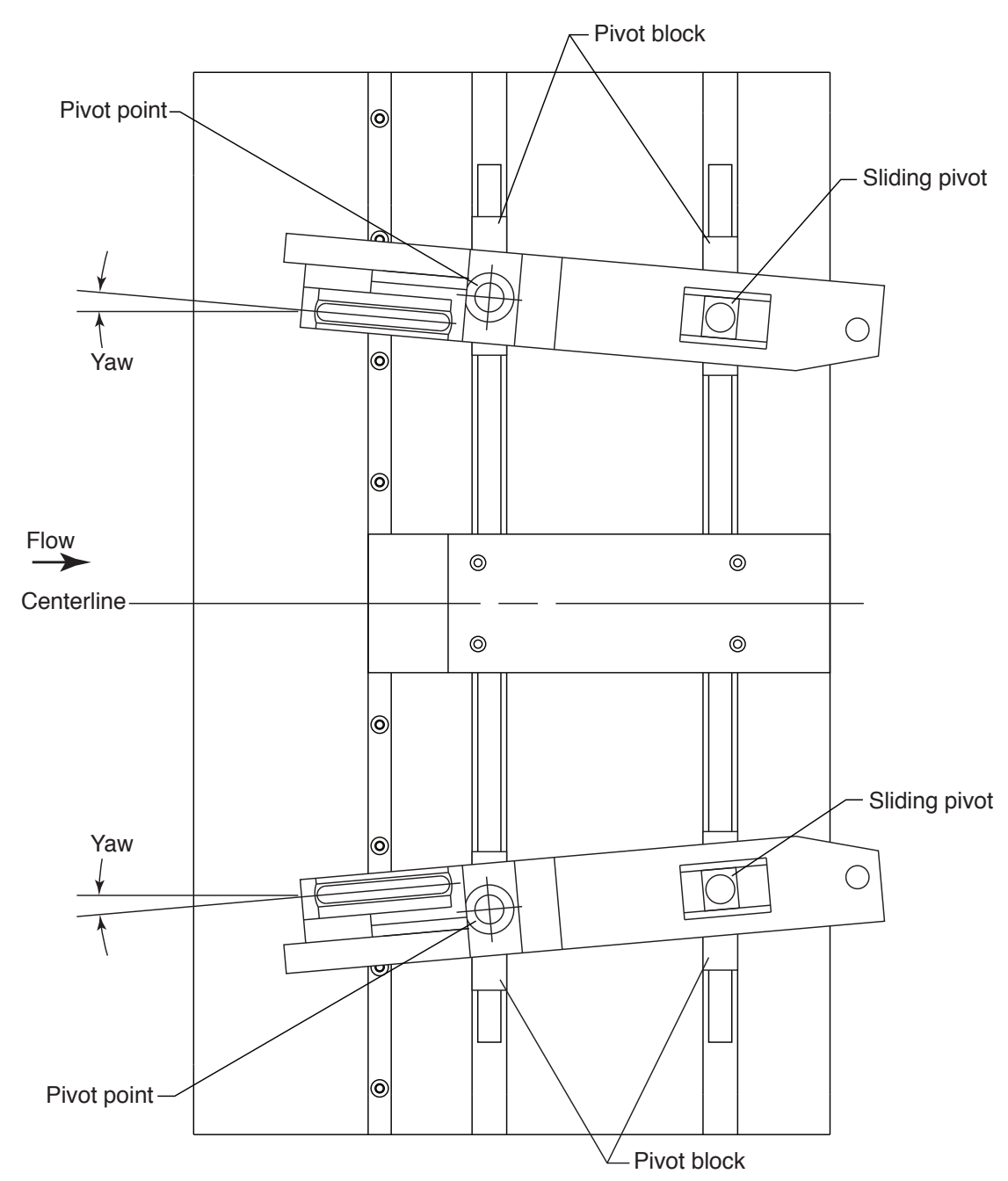

(b) Schematic of sting brackets at yaw.

Figure 9. Separation rig. 


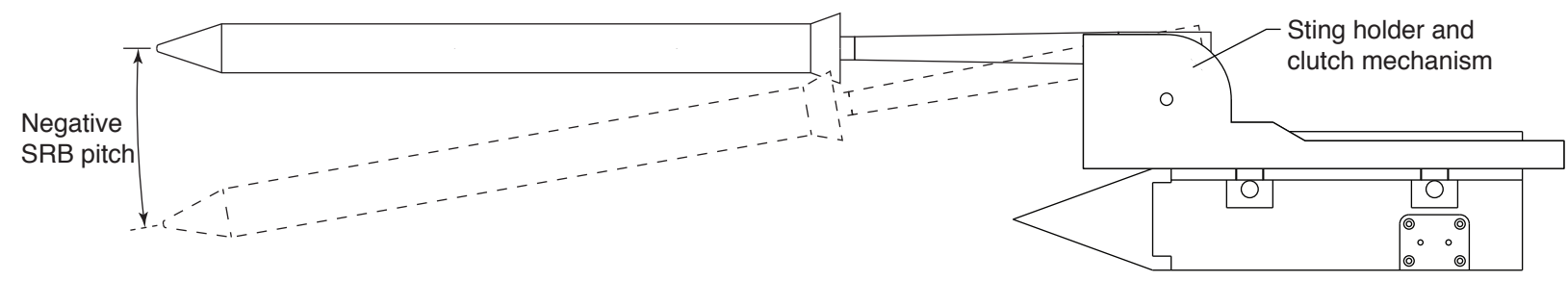

Figure 10. SRB pitch illustration.

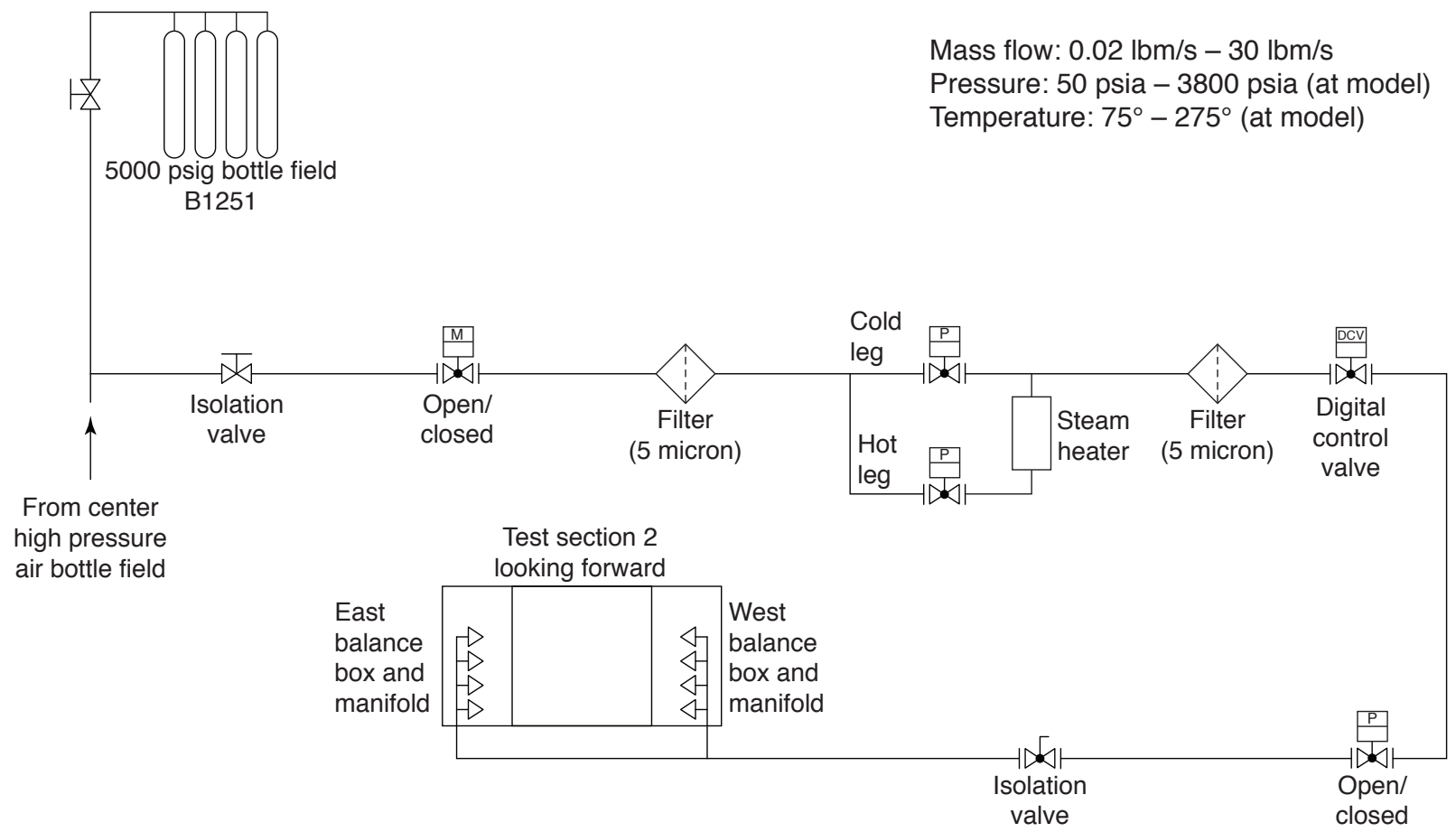

Figure 11. Simplified schematic of UPWT high-pressure air system. 


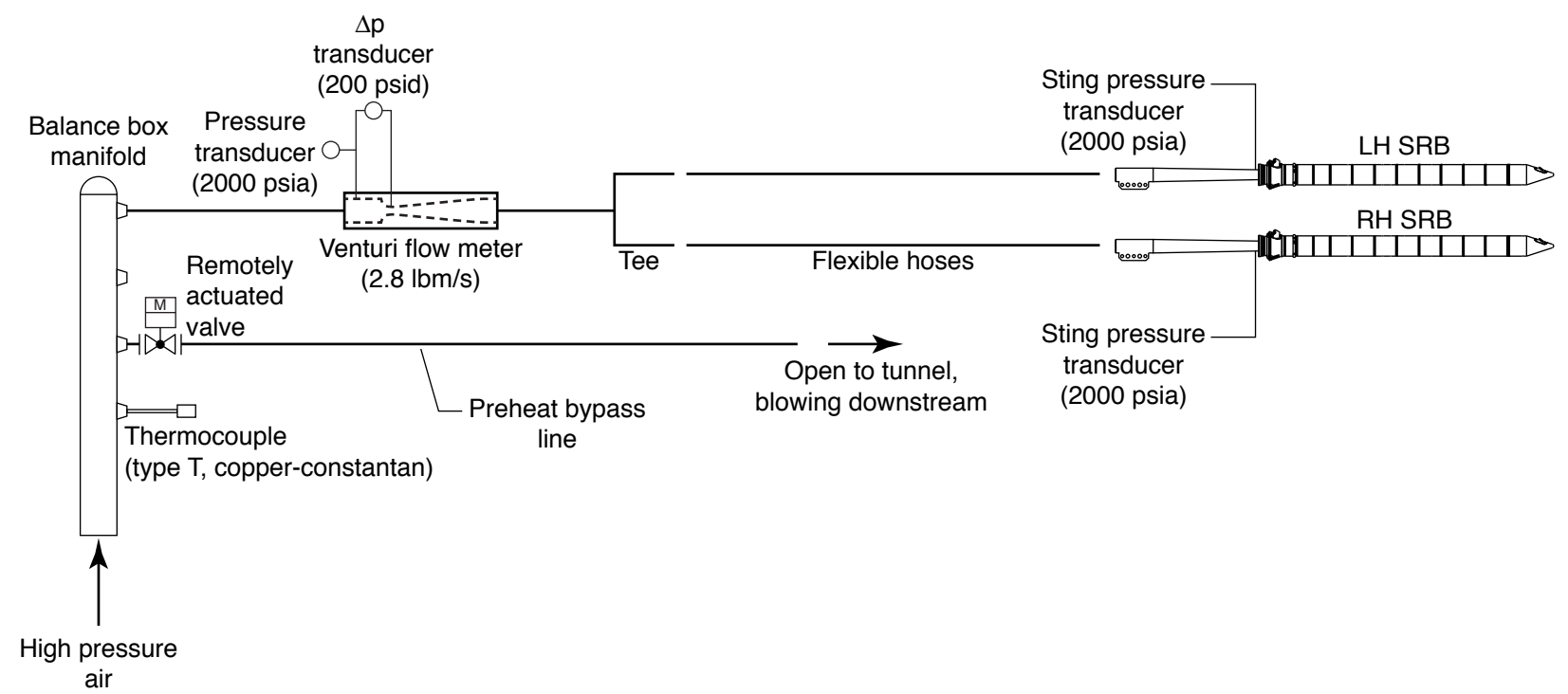

Figure 12. Schematic of high-pressure air routing from balance box manifold to SRB.

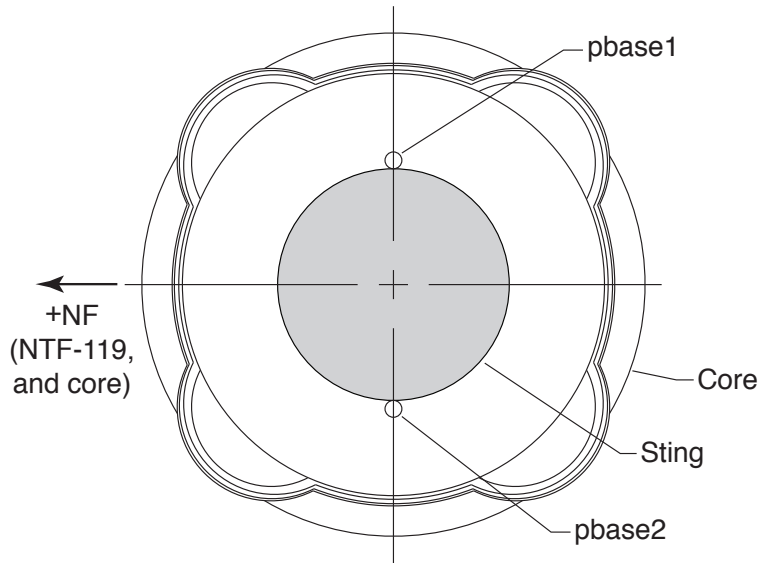

Chamber pressure tubes extend approx. 4 in. into aft end of model

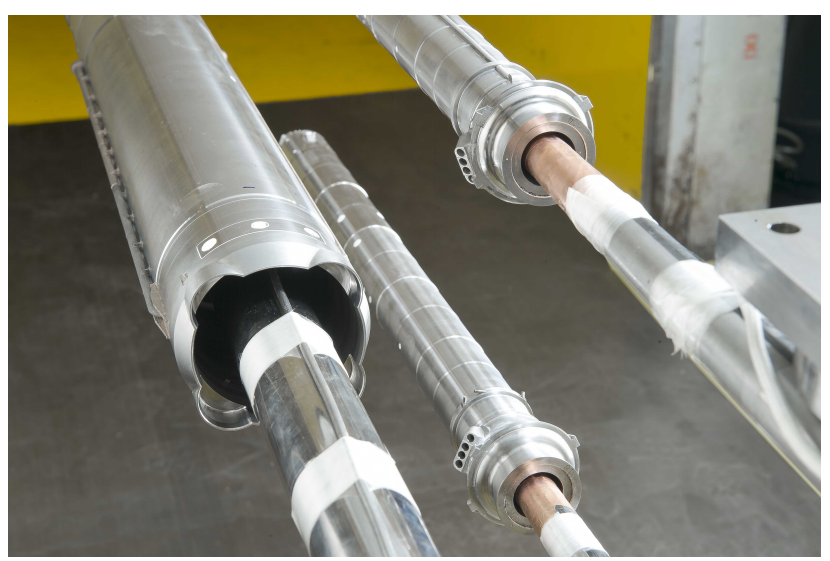

(b) View looking upstream.

(a) Location of chamber pressure tubes.

Figure 13. Core chamber pressure tubes. 


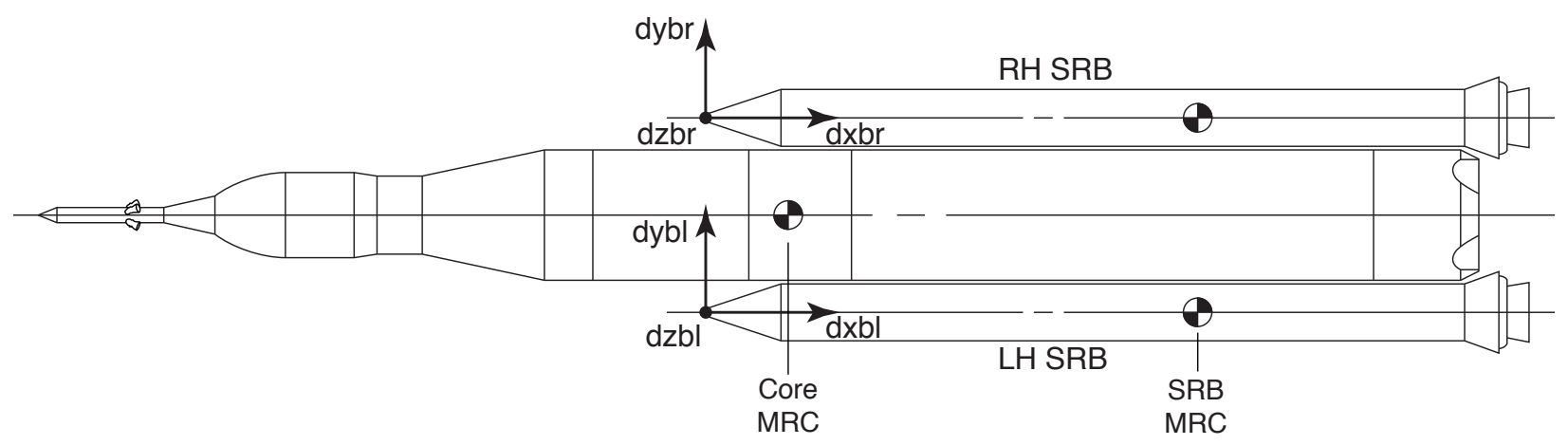

Top view

Figure 14. Orientation and location of delta nose position origin (dzbr and dzbl are pointing out of page). 


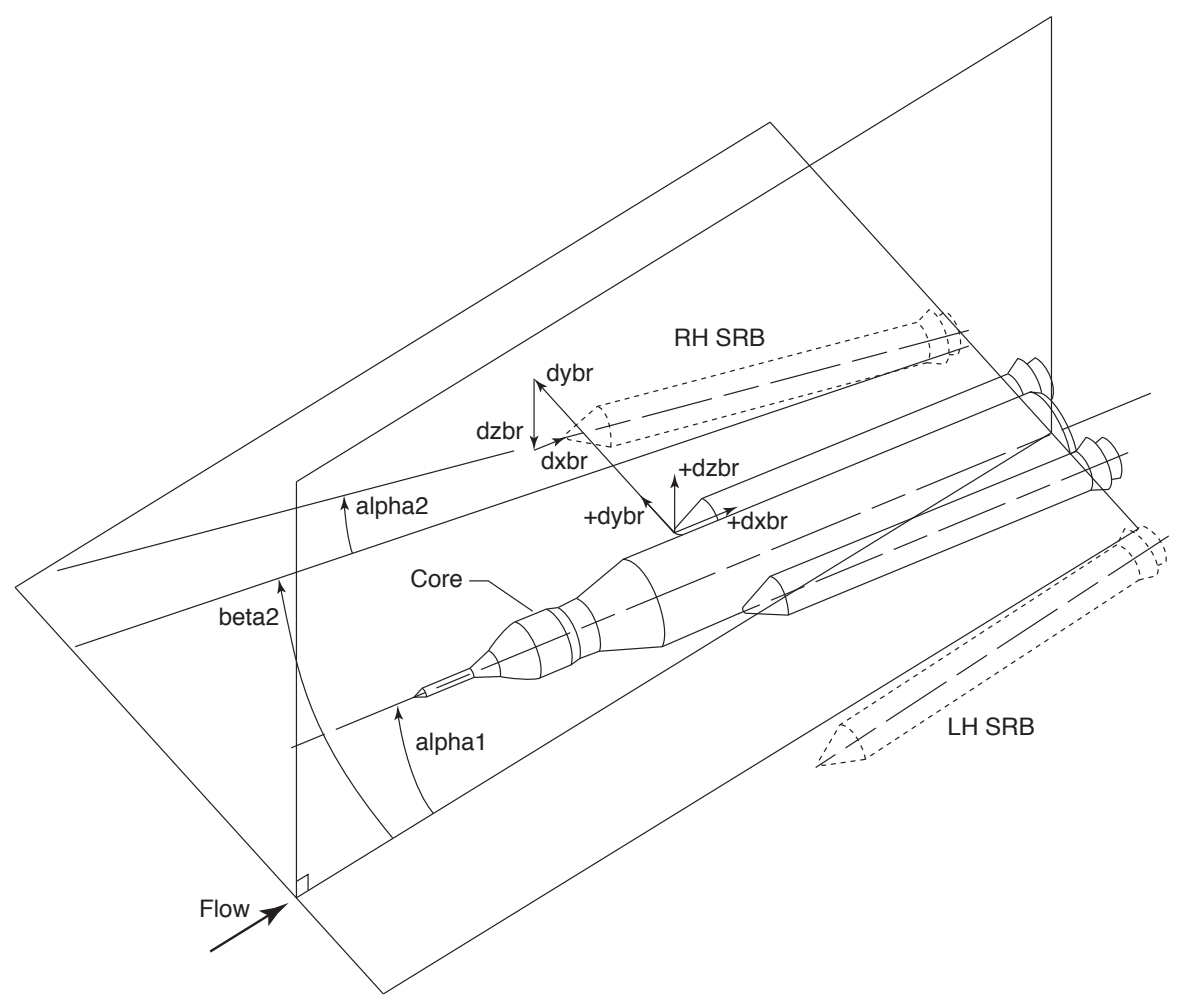

Figure 15. Delta nose position of RH SRB from launch configuration.

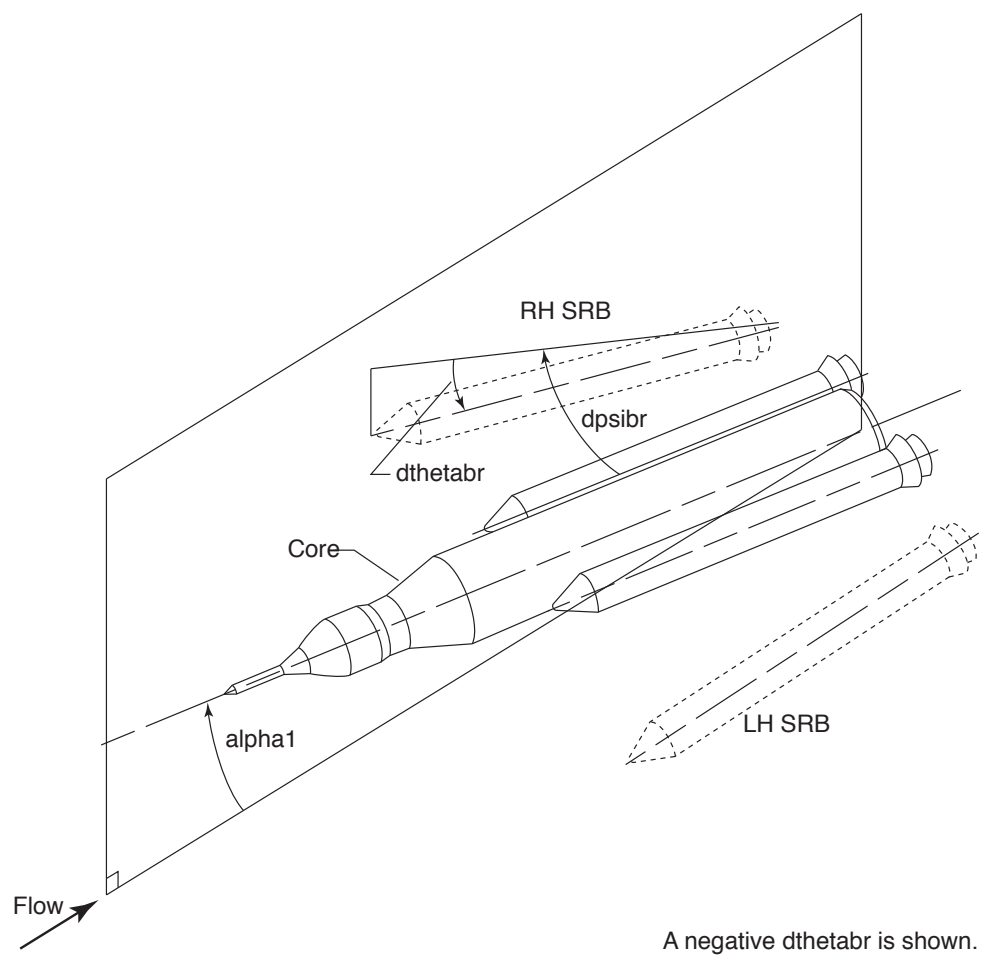

Figure 16. Delta angular position of RH SRB from launch configuration. 


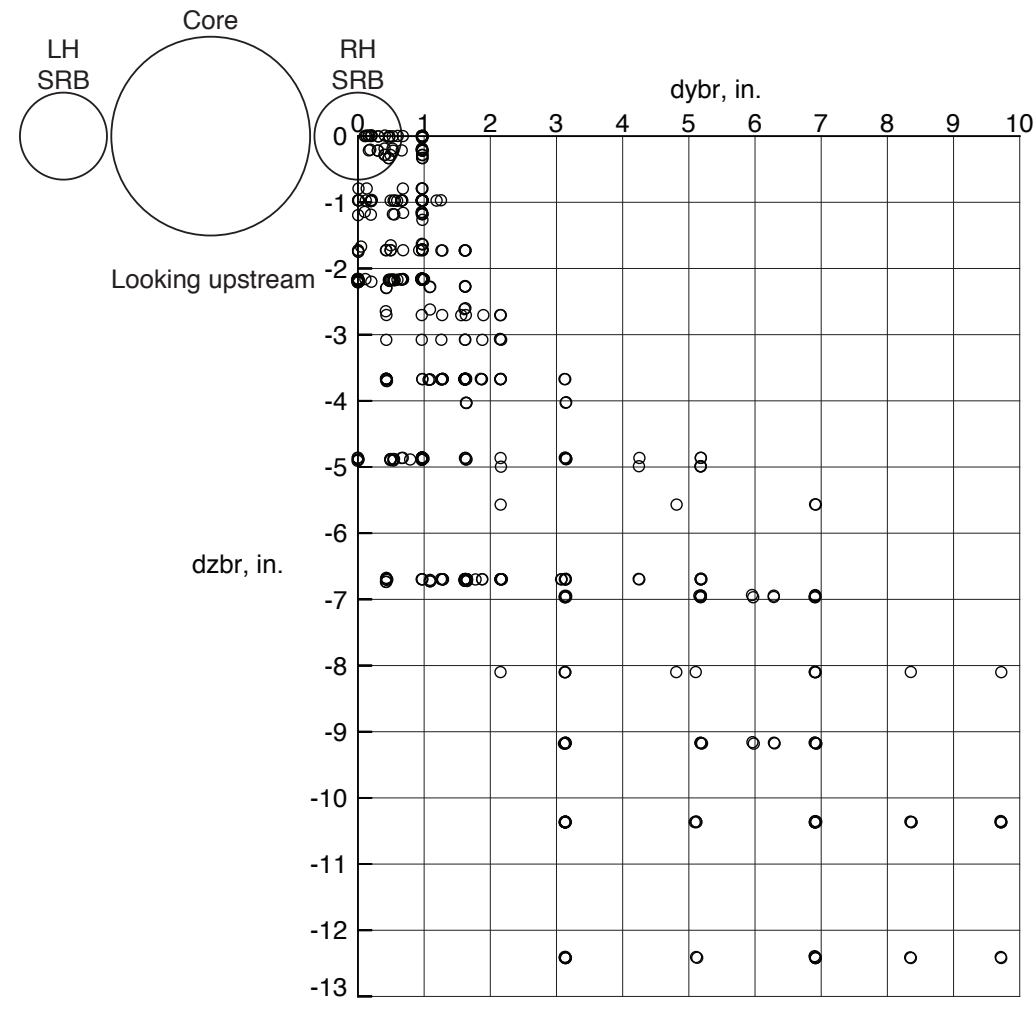

Figure 17. All RH SRB nose positions tested.

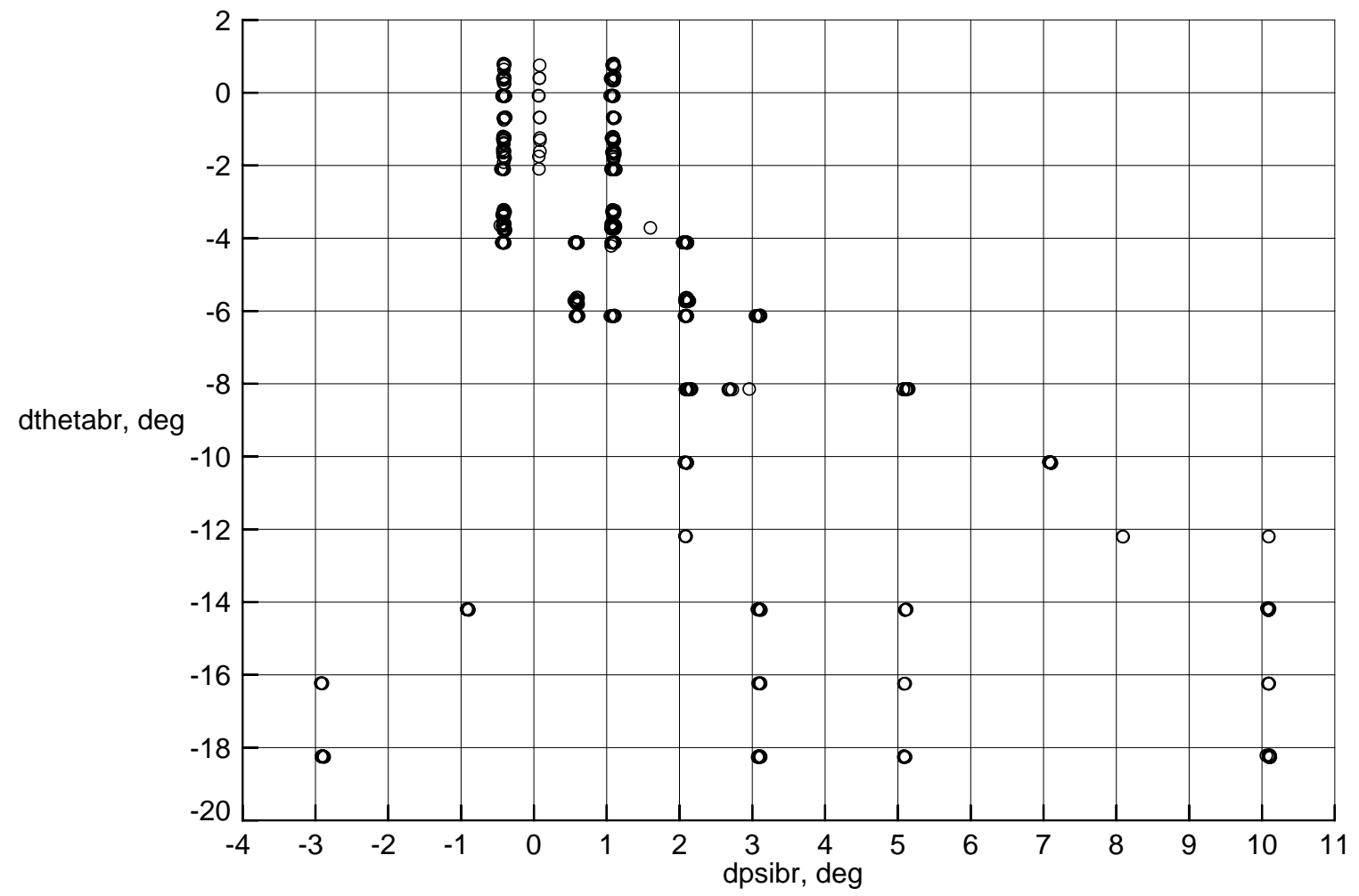

Figure 18. All RH SRB nose attitudes tested. 


$\begin{array}{ccccccccc} & \text { Run } & \text { Mach } & \text { dybr, in. } & \text { dzbr, in. } & \text { dpsibr, deg } & \text { dthetabr, deg } & \text { pstingbr, psia } & \text { Data type } \\ \circ & 490 & 4.25 & 0.95 & -1.98 & -0.43 & -3.62 & 1381.0 & \text { continuous } \\ \square & 491 & 4.25 & 0.95 & -2.00 & -0.43 & -3.62 & 1380.3 & \text { move pause }\end{array}$
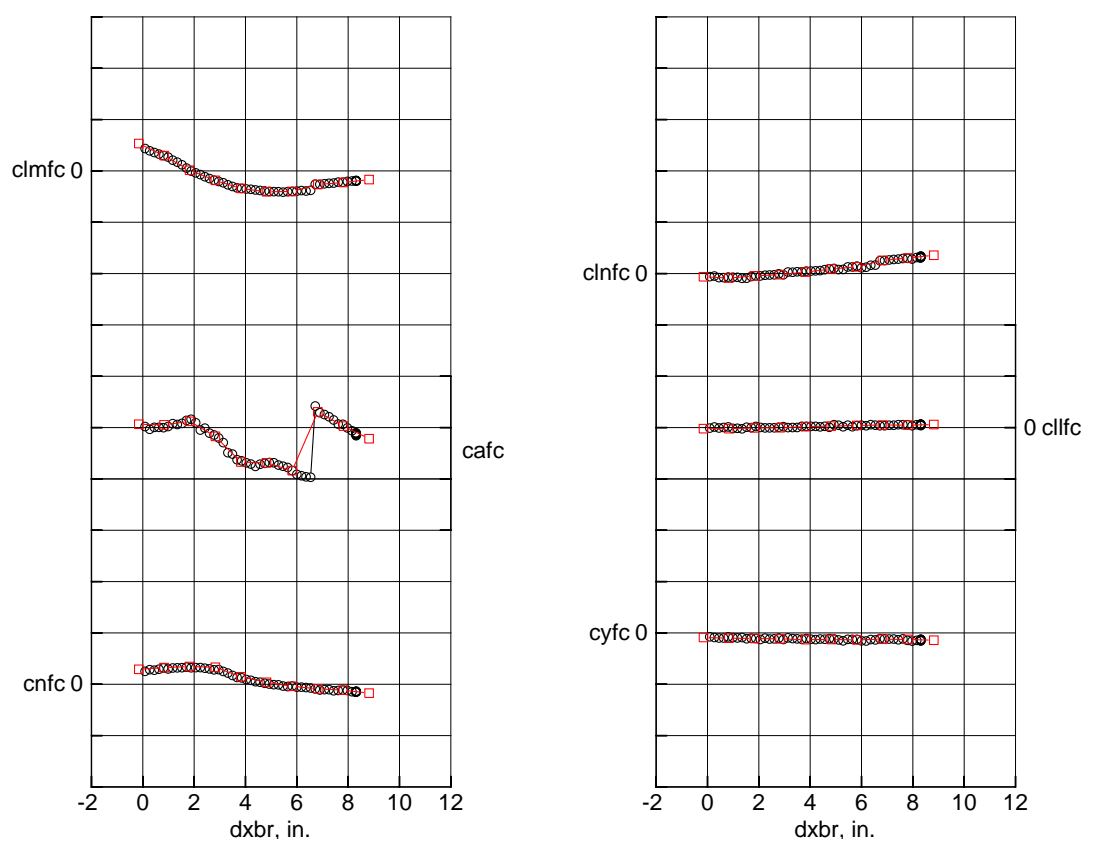

Figure 19. Typical core aerodynamics with BSM plume simulation when core nose shock jumps from LAS tip to capsule.

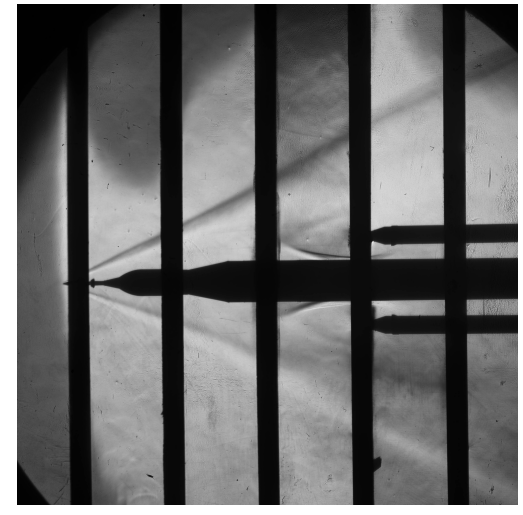

(a) $d x b r=6$ in

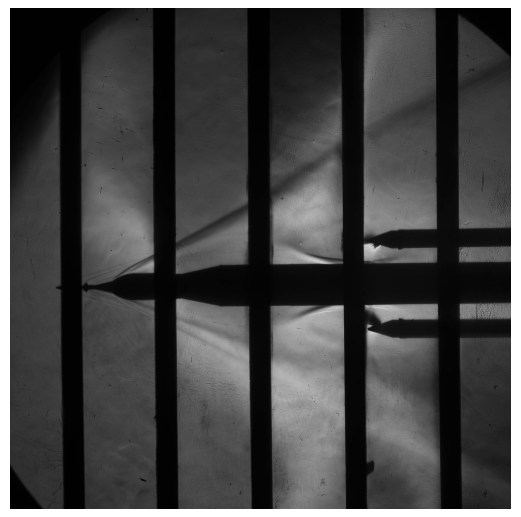

(b) $d x b r=7$ in

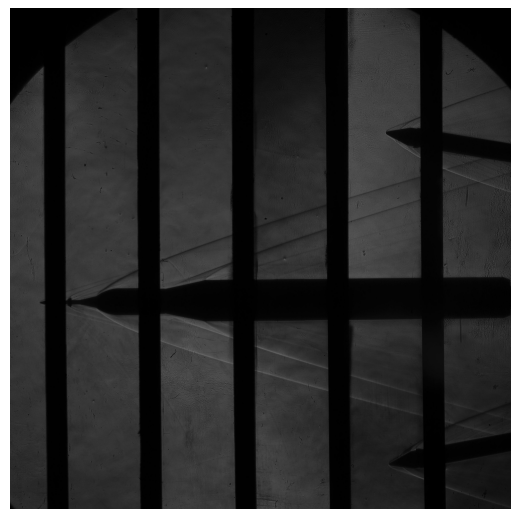

(c) Core alone.

Figure 20. Schlieren images showing core nose shock jump from LAS tip to capsule with BSM plume simulation and core alone flow field. 


\begin{tabular}{ccccccccc} 
& Run & Mach & dybr, in. & dzbr, in. & dpsibr, deg & dthetabr, deg & pstingbr, psia & Data type \\
\hline & 284 & 4.25 & 0.43 & -3.68 & 0.60 & -5.71 & 1381.2 & continuous
\end{tabular}
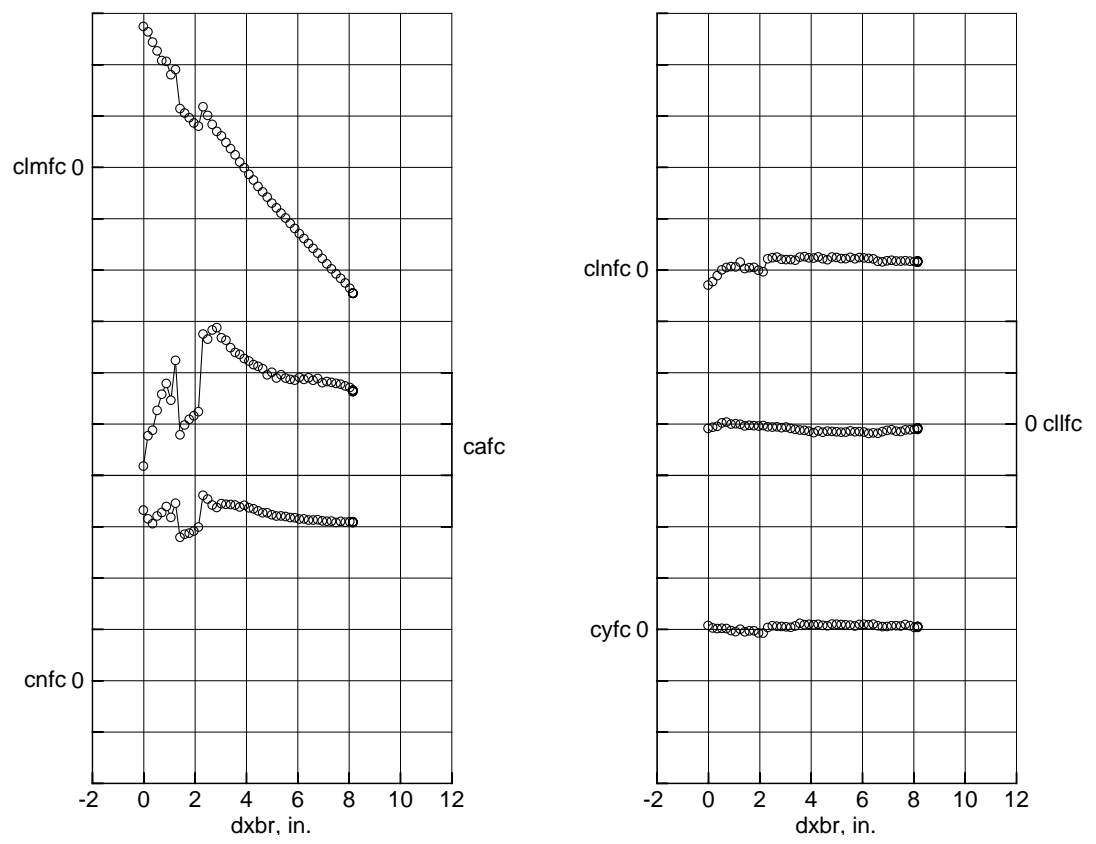

Figure 21. Core aerodynamics when core nose shock was jumping between the LAS tip and capsule. 
LAS

Jumping

Capsule

All BSM nozzles open

Orange box indicates separation trajectory bounds from GN\&C simulation (limited to plot range)

Dashed orange box indicates BSMs not firing (BSMs fire for approximately $1 \mathrm{~s}$ )
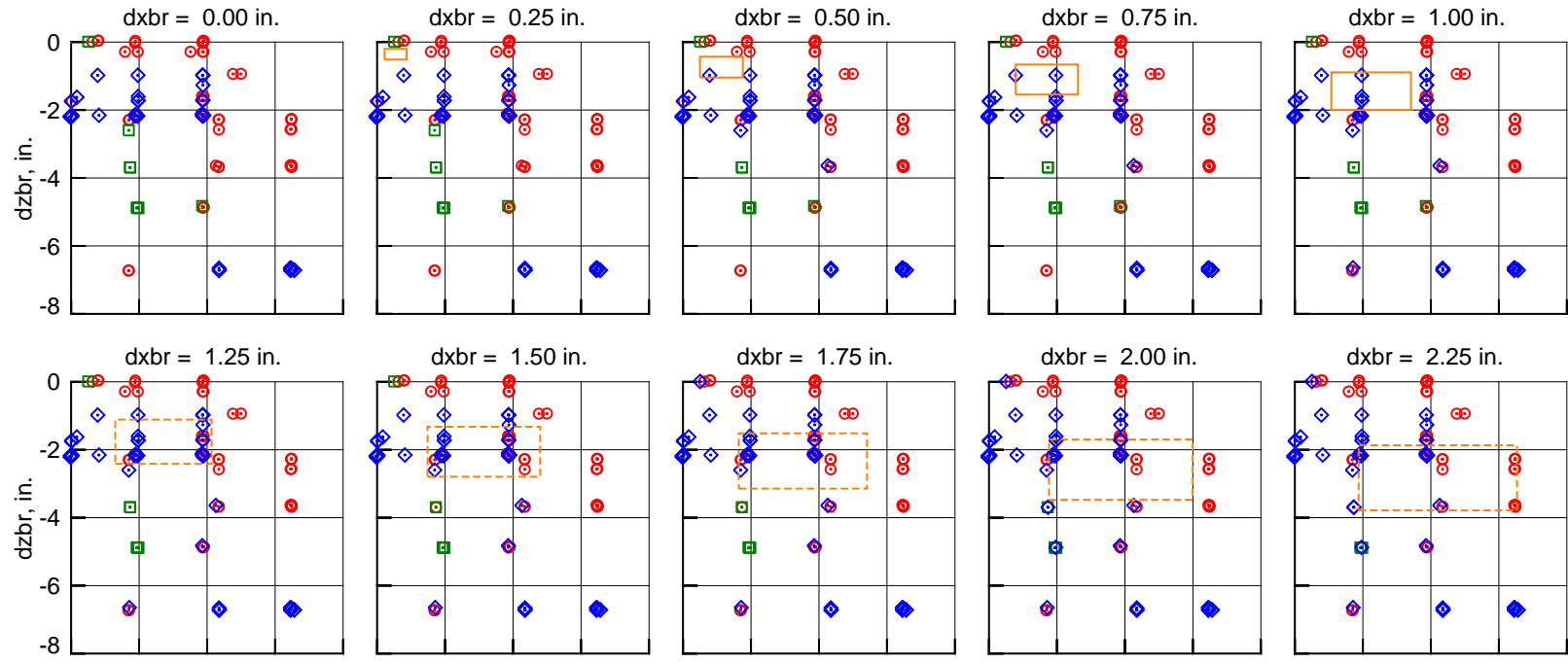

$\mathrm{dxbr}=1.50 \mathrm{in}$

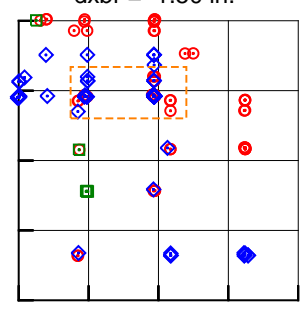

$\mathrm{dxbr}=1.75 \mathrm{in}$

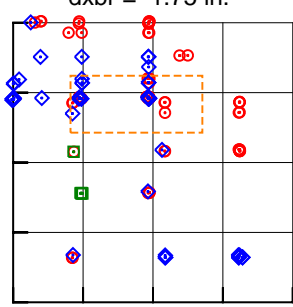

$\mathrm{dxbr}=2.00 \mathrm{in}$.

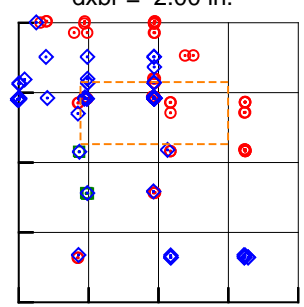

$\mathrm{dxbr}=2.25 \mathrm{in}$
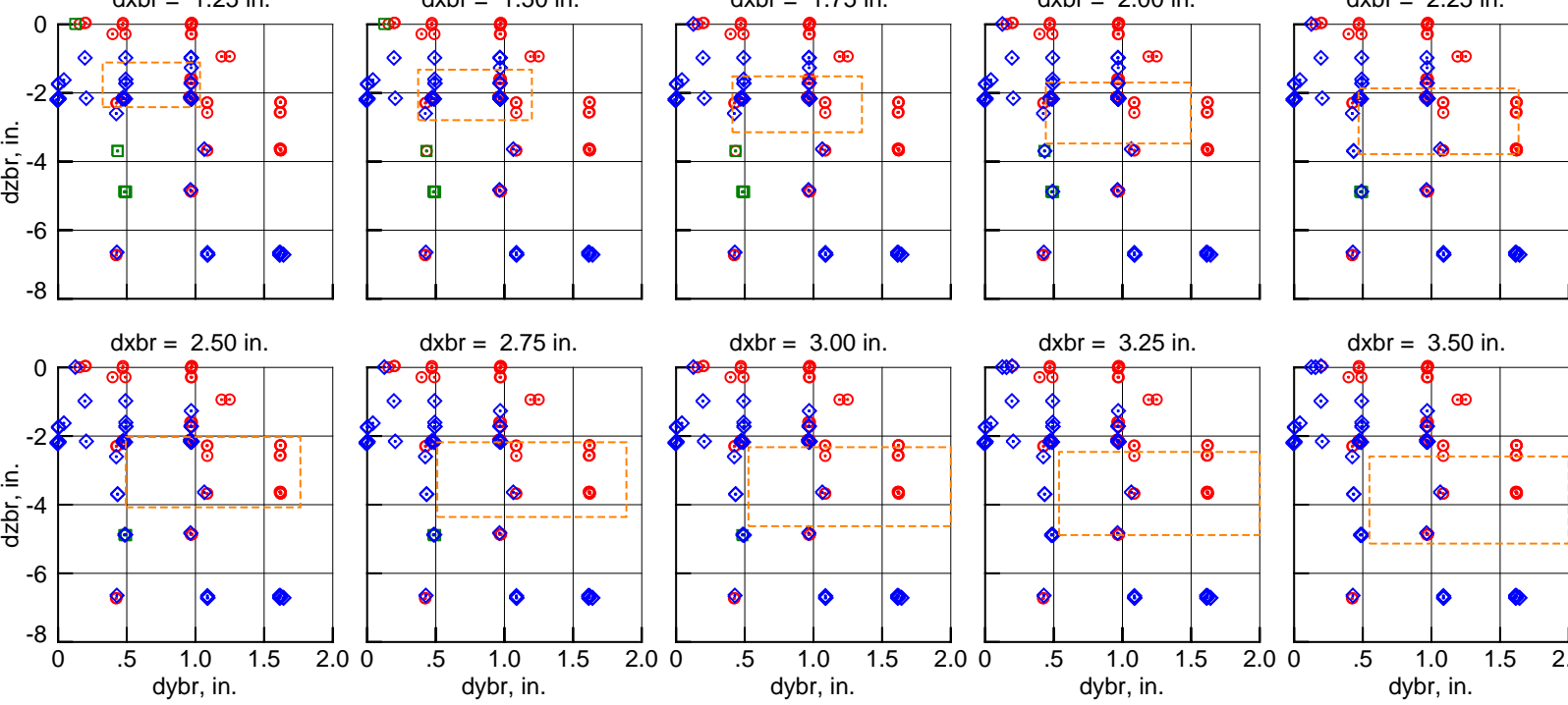

Figure 22. Core nose shock location.

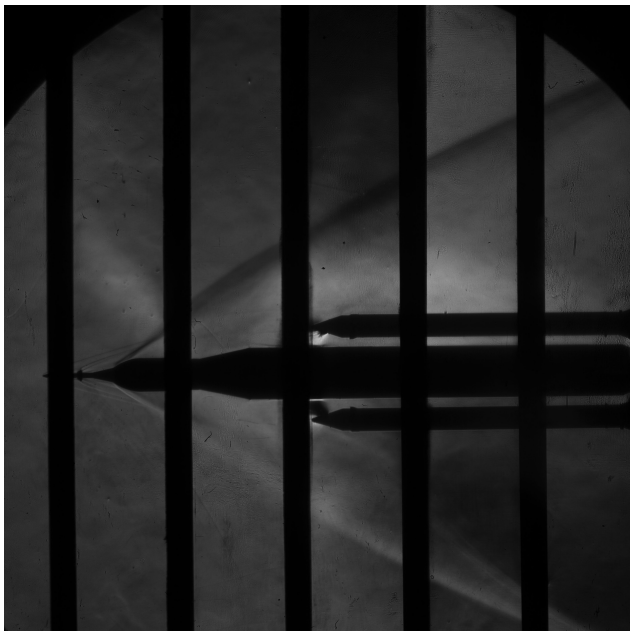

(a) pstingbr $\approx 1100$ psia.

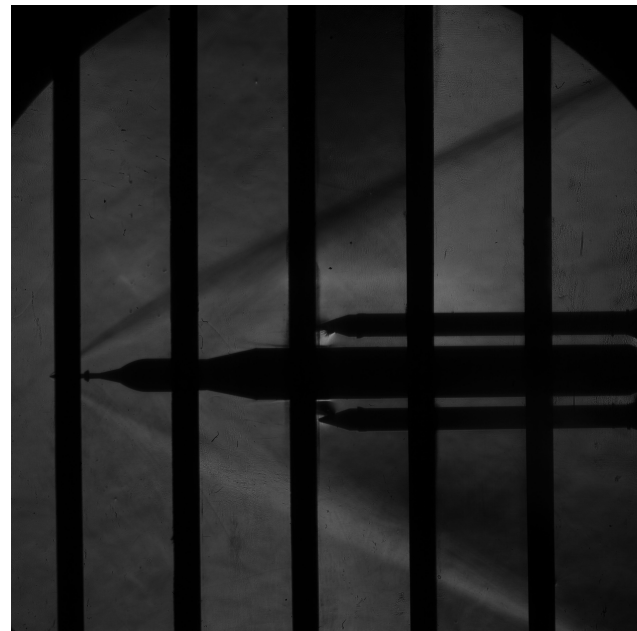

(b) pstingbr $\approx 1200$ psia.

Figure 23. Schlieren images showing core nose shock jump from capsule to LAS tip as BSM plenum pressure is increased. 

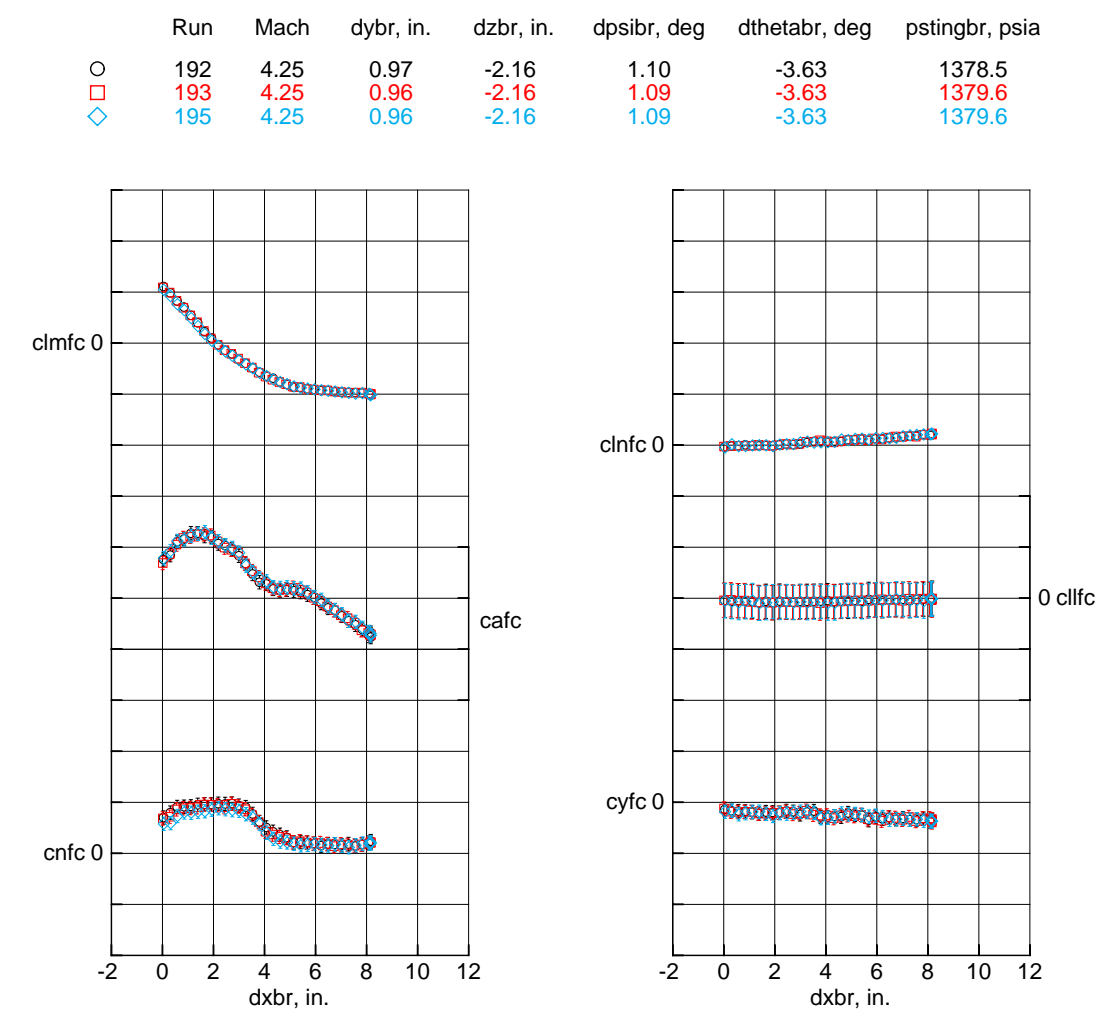

(a) Core.
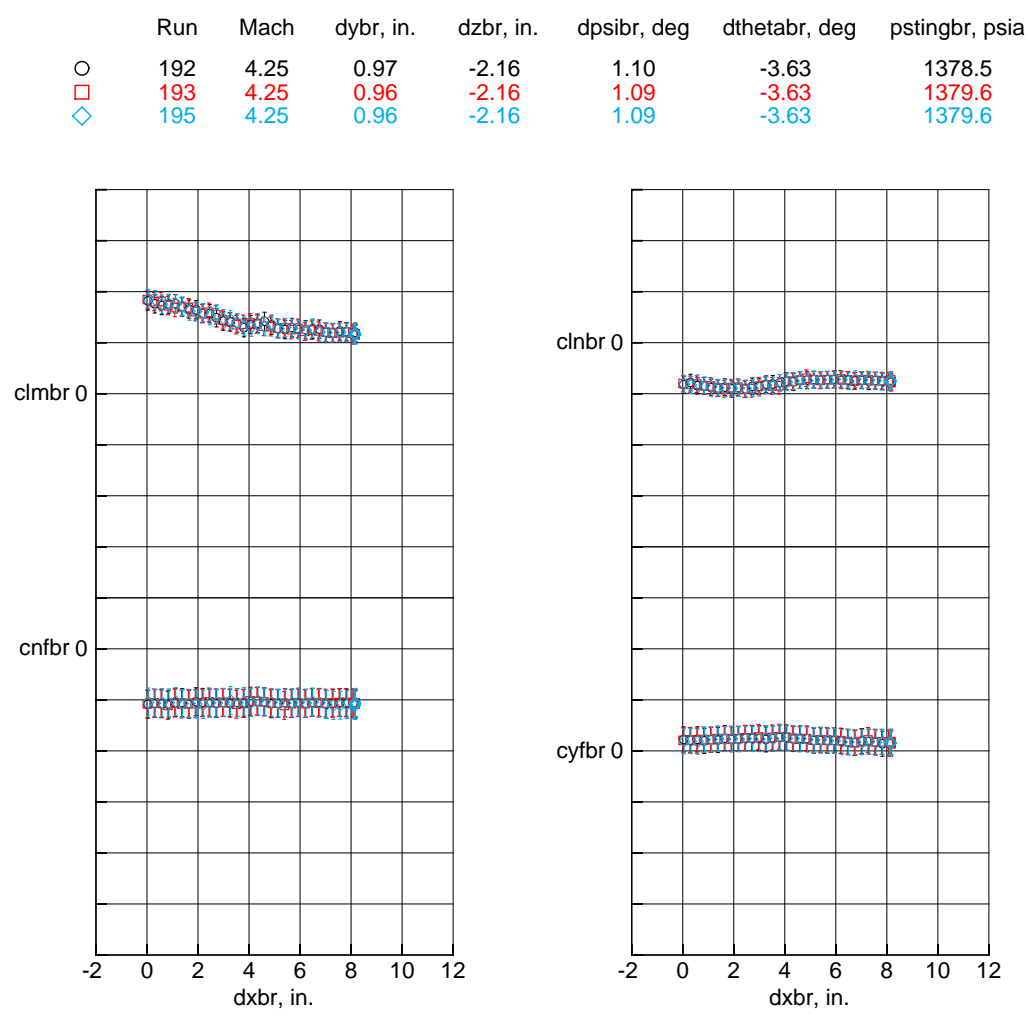

(b) RH SRB.

Figure 24. First set of back-to-back repeat runs for the core and SRBs with BSM plume simulation. Error bars represent balance accuracy; plotting every 30th frame of continuous data. 


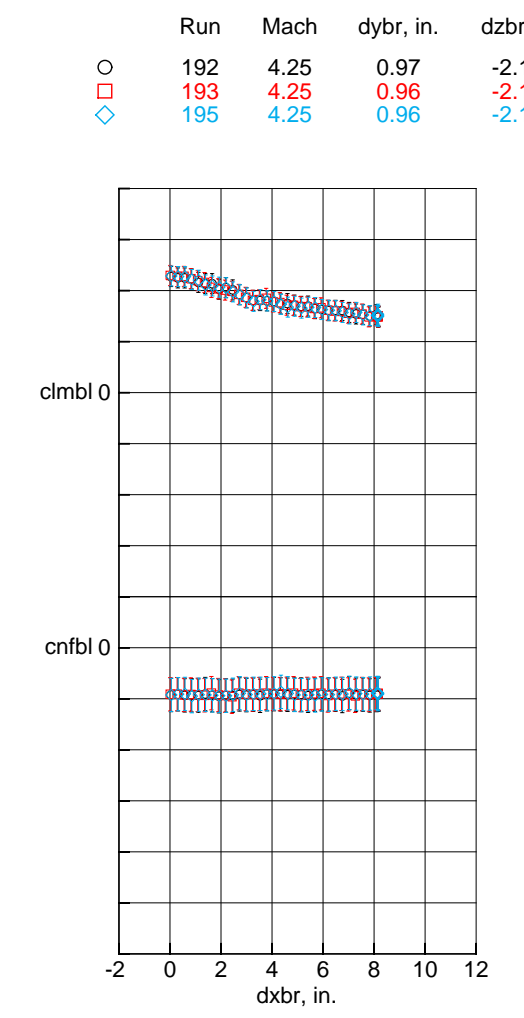

$\begin{array}{ccc}\text { dpsibr, deg } & \text { dthetabr, deg } & \text { pstingbr, psia } \\ 1.10 & -3.63 & 1378.5 \\ 1.09 & -3.63 & 1379.6 \\ 1.09 & -3.63 & 1379.6\end{array}$

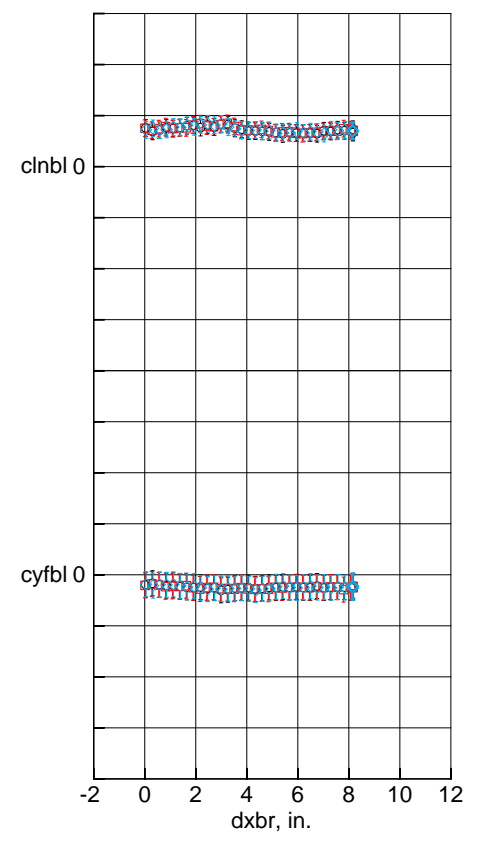

(c) LH SRB.

Figure 24. Concluded. 

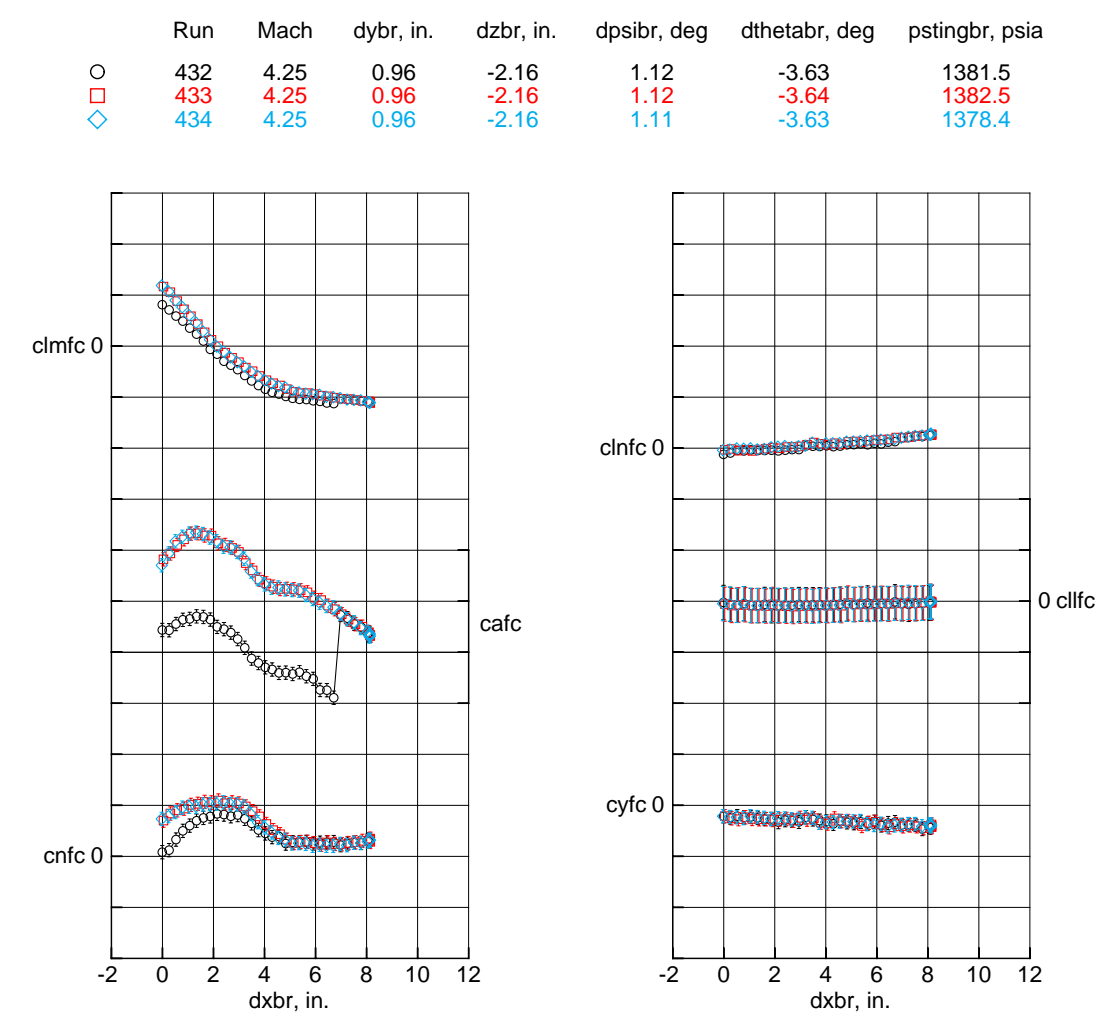

(a) Core.

$\begin{array}{cccccccc} & \text { Run } & \text { Mach } & \text { dybr, in. } & \text { dzbr, in. } & \text { dpsibr, deg } & \text { dthetabr, deg } & \text { pstingbr, psia } \\ \circ & 432 & 4.25 & 0.96 & -2.16 & 1.12 & -3.63 & 1381.5 \\ \triangleright & 433 & 4.25 & 0.96 & -2.16 & 1.12 & -3.64 & 1382.5 \\ \diamond & 434 & 4.25 & 0.96 & -2.16 & 1.11 & -3.63 & 1378.4\end{array}$
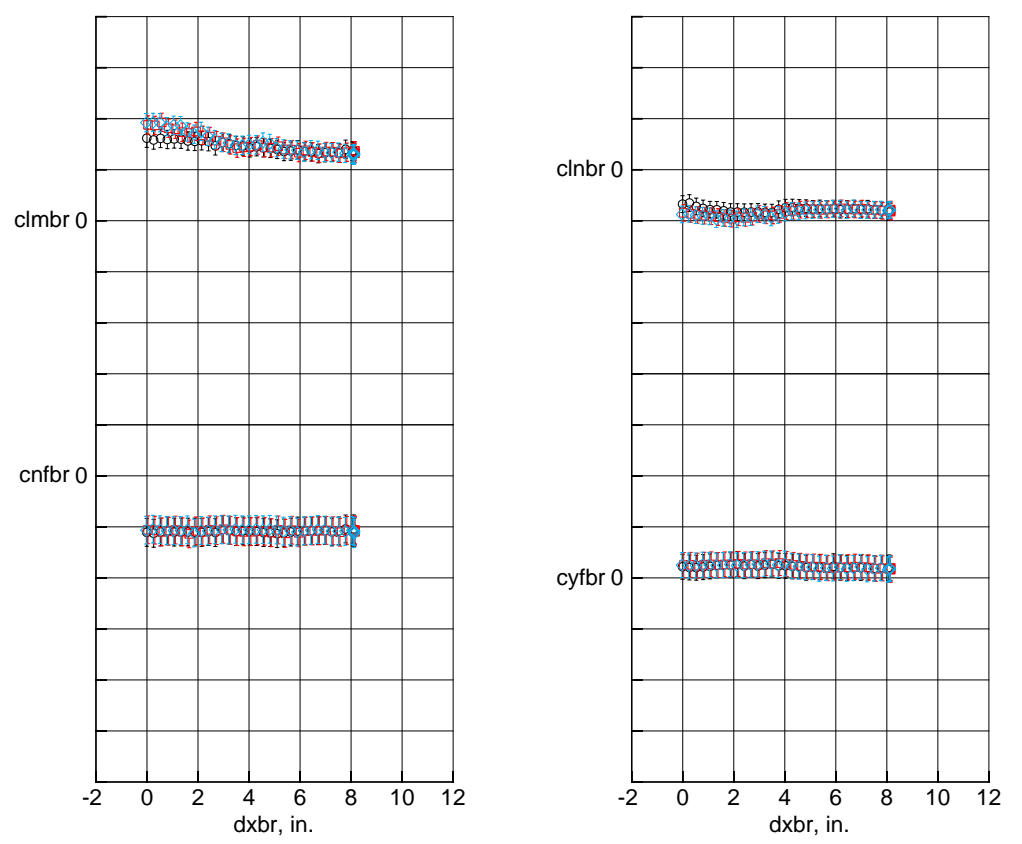

(b) RH SRB.

Figure 25. Third set of back-to-back repeat runs for the core and SRBs with BSM plume simulation. Error bars represent balance accuracy; plotting every 30th frame of continuous data. 


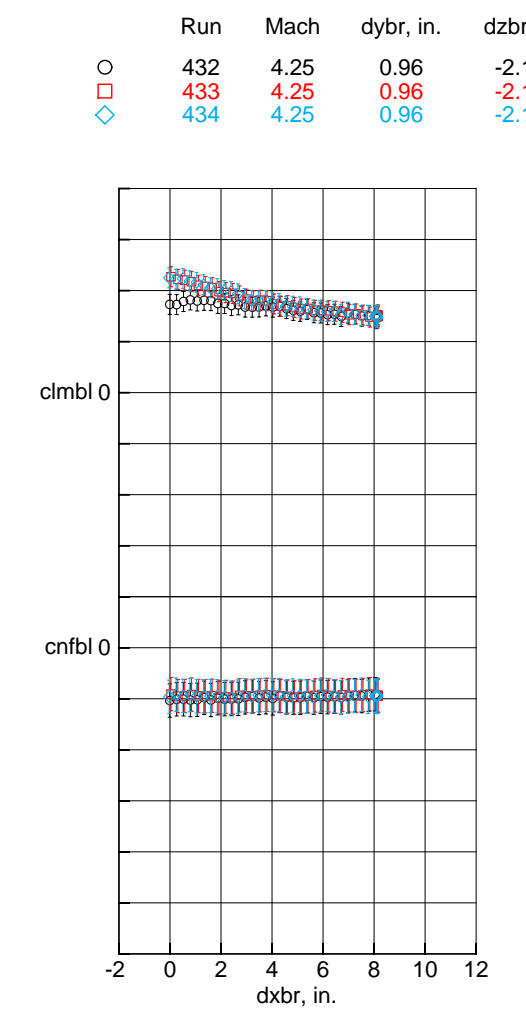

$\begin{array}{ccc}\text { dpsibr, deg } & \text { dthetabr, deg } & \text { pstingbr, psia } \\ 1.12 & -3.63 & 1381.5 \\ 1.12 & -3.64 & 1382.5 \\ 1.11 & -3.63 & 1378.4\end{array}$

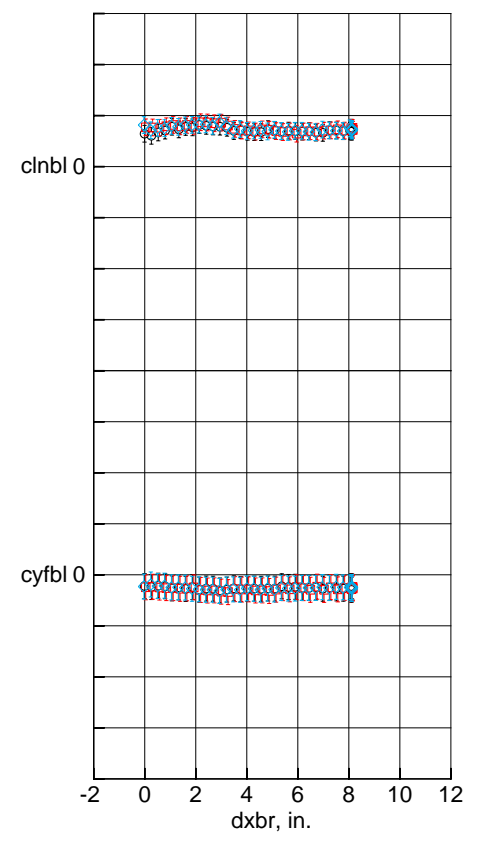

(c) LH SRB.

Figure 25. Concluded. 
Run Mach dybr, in. dzbr, in. dpsibr, deg dthetabr, deg pstingbr, psia

$\begin{array}{lllllll}144 & 4.25 & 1.61 & -6.70 & 2.11 & -4.13 & \text { off }\end{array}$

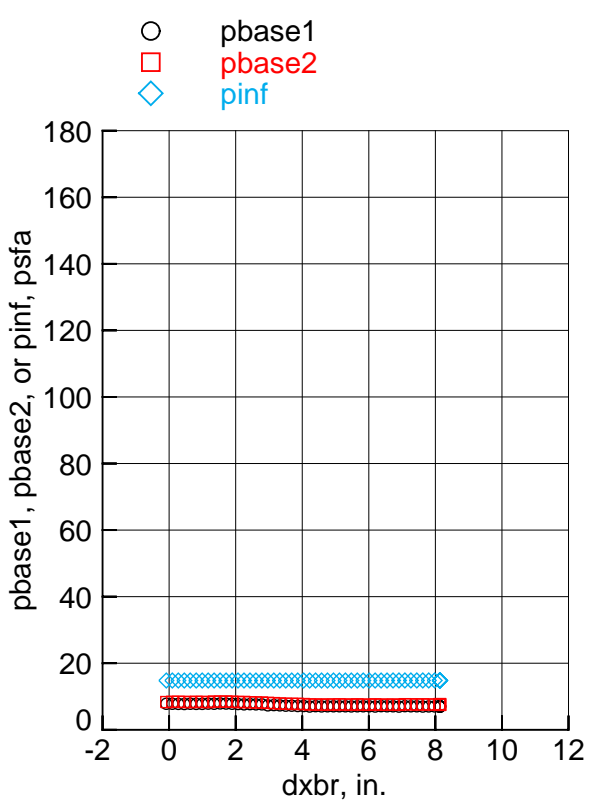

(a) $d z b r=-6.70$ in.
Run Mach dybr, in. dzbr, in. dpsibr, deg dthetabr, deg pstingbr, psia

$\begin{array}{lllllll}155 & 4.25 & 1.61 & -3.67 & 2.10 & -4.11 & \text { off }\end{array}$

○ pbase1

$\checkmark \quad \begin{aligned} & \square \text { pinf } \\ & \square \quad \text { pine2 }\end{aligned}$

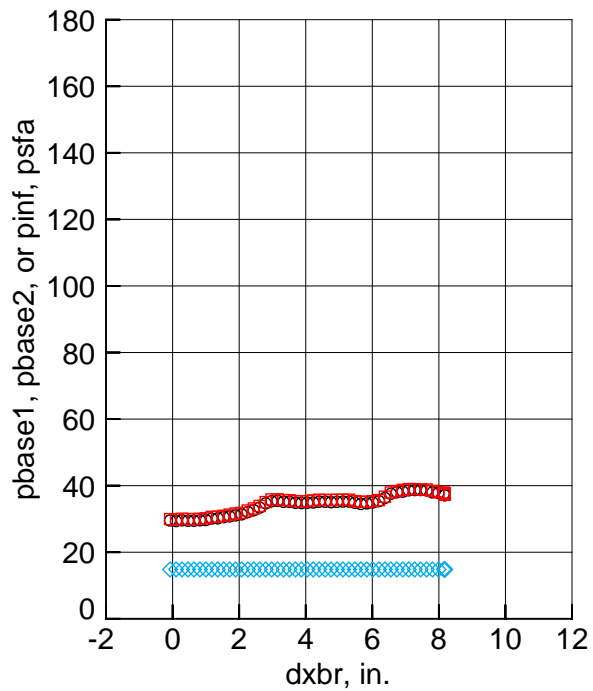

(b) $d z b r=-3.67$ in.

Figure 26. Core chamber pressures without BSM plume simulation.

Run Mach dybr, in. dzbr, in. dpsibr, deg dthetabr, deg pstingbr, psia

$\begin{array}{lllllll}279 & 4.25 & 1.61 & -6.70 & 2.08 & -5.69 & 1380.4\end{array}$

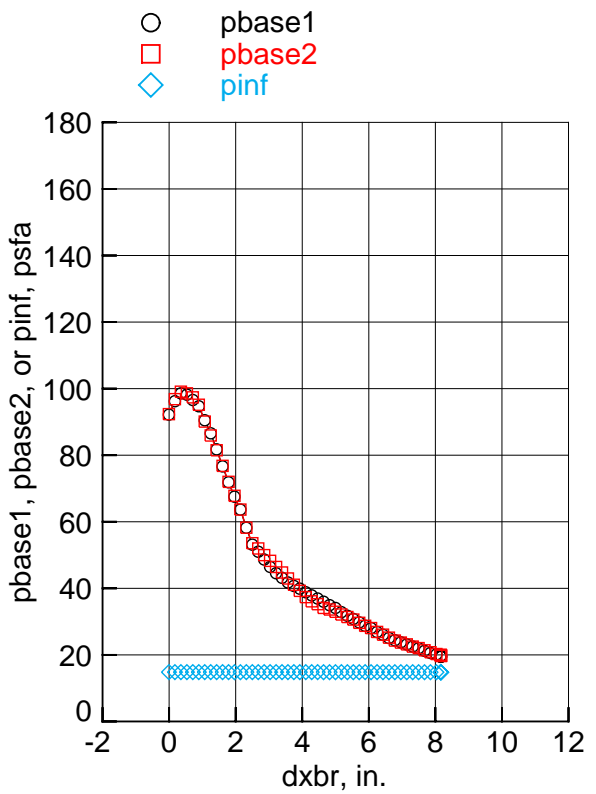

(a) $d z b r=-6.70$ in.
Run Mach dybr, in. dzbr, in. dpsibr, deg dthetabr, deg pstingbr, psia

$\begin{array}{lllllll}286 & 4.25 & 1.61 & -3.67 & 2.11 & -5.67 & 1384.2\end{array}$

$\square \quad$ pbase2

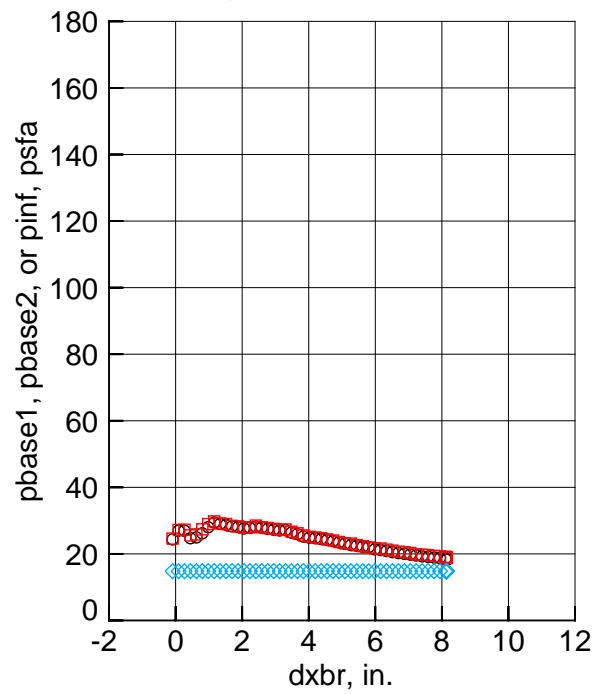

(b) $d z b r=-3.67 \mathrm{in}$.

Figure 27. Core chamber pressures with BSM plume simulation. 


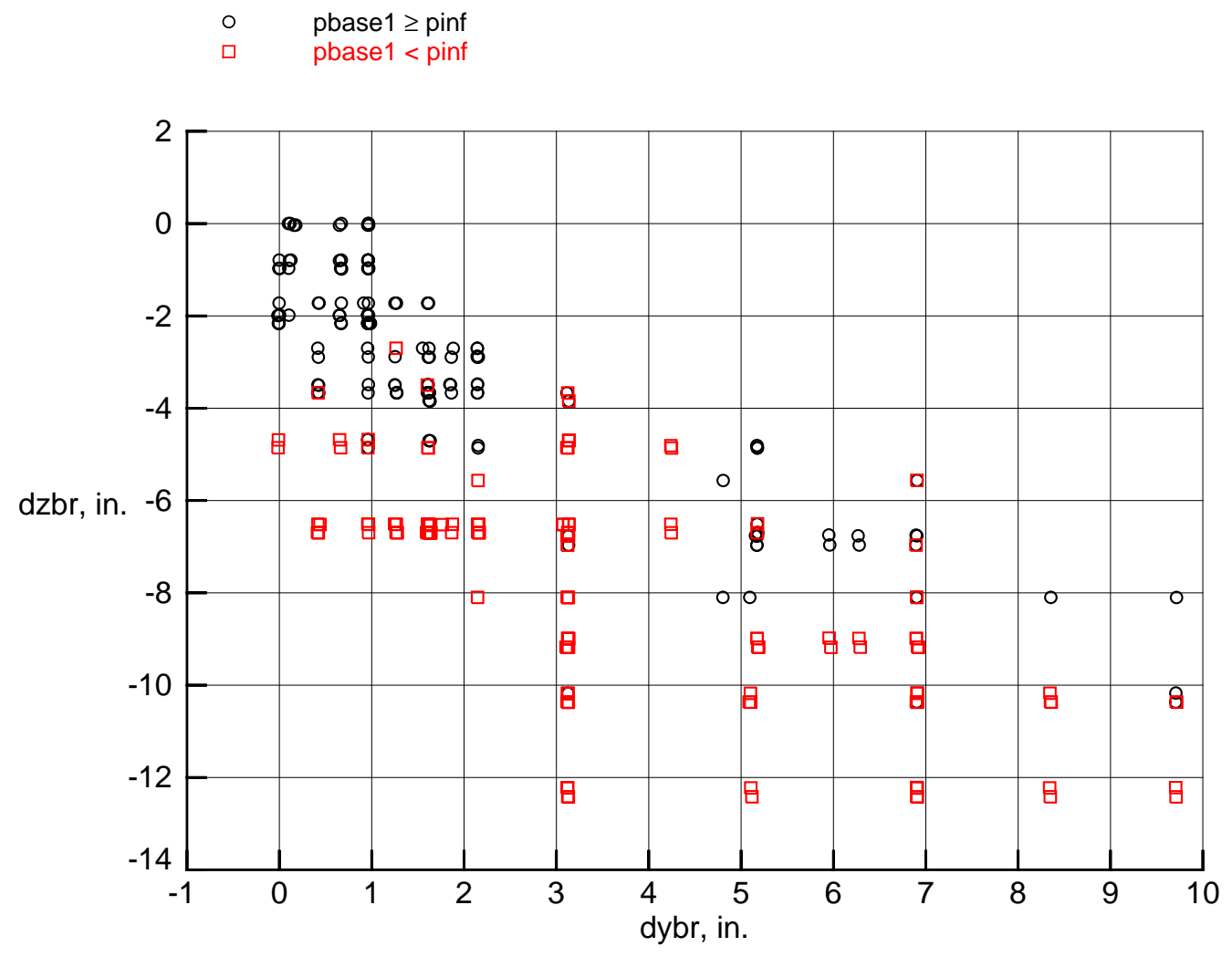

Figure 28. Runs without BSM plume simulation where core chamber pressure is above or below free-stream static pressure.

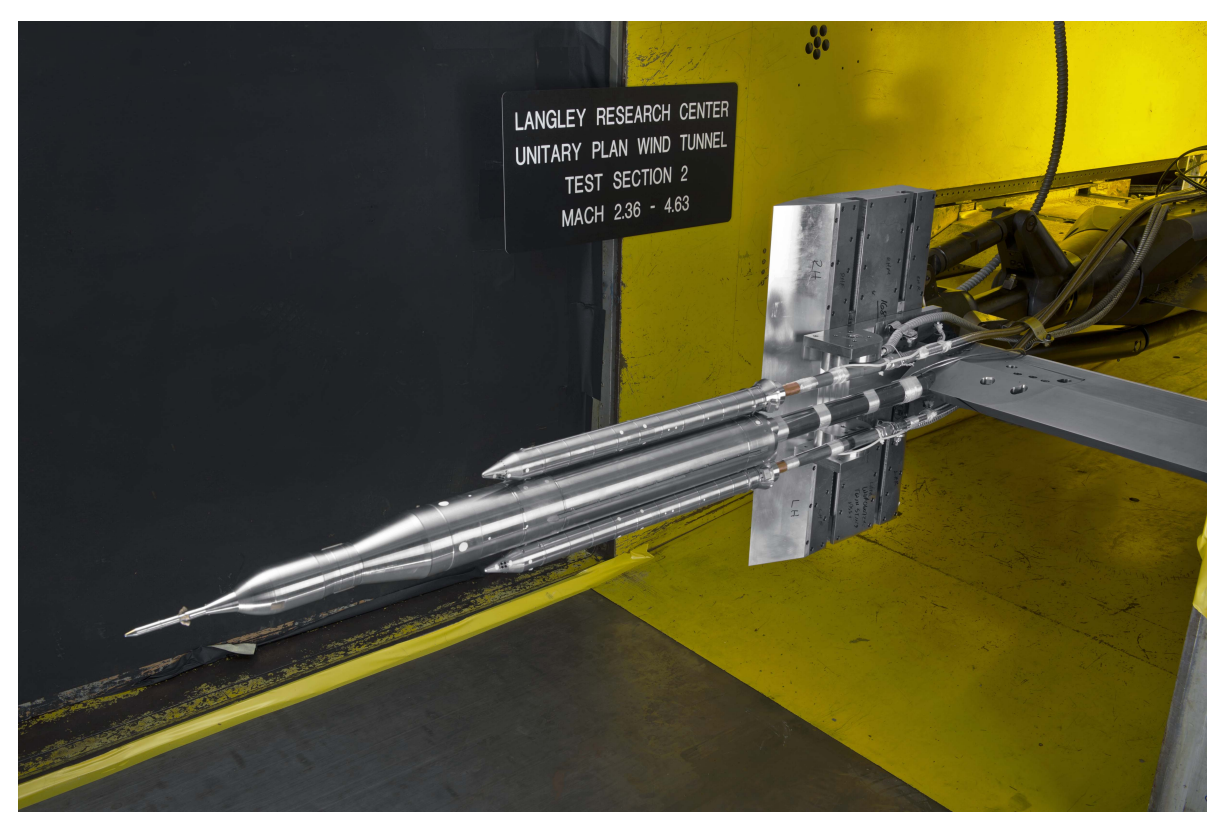

Figure 29. View of separation rig close to core support strut. 


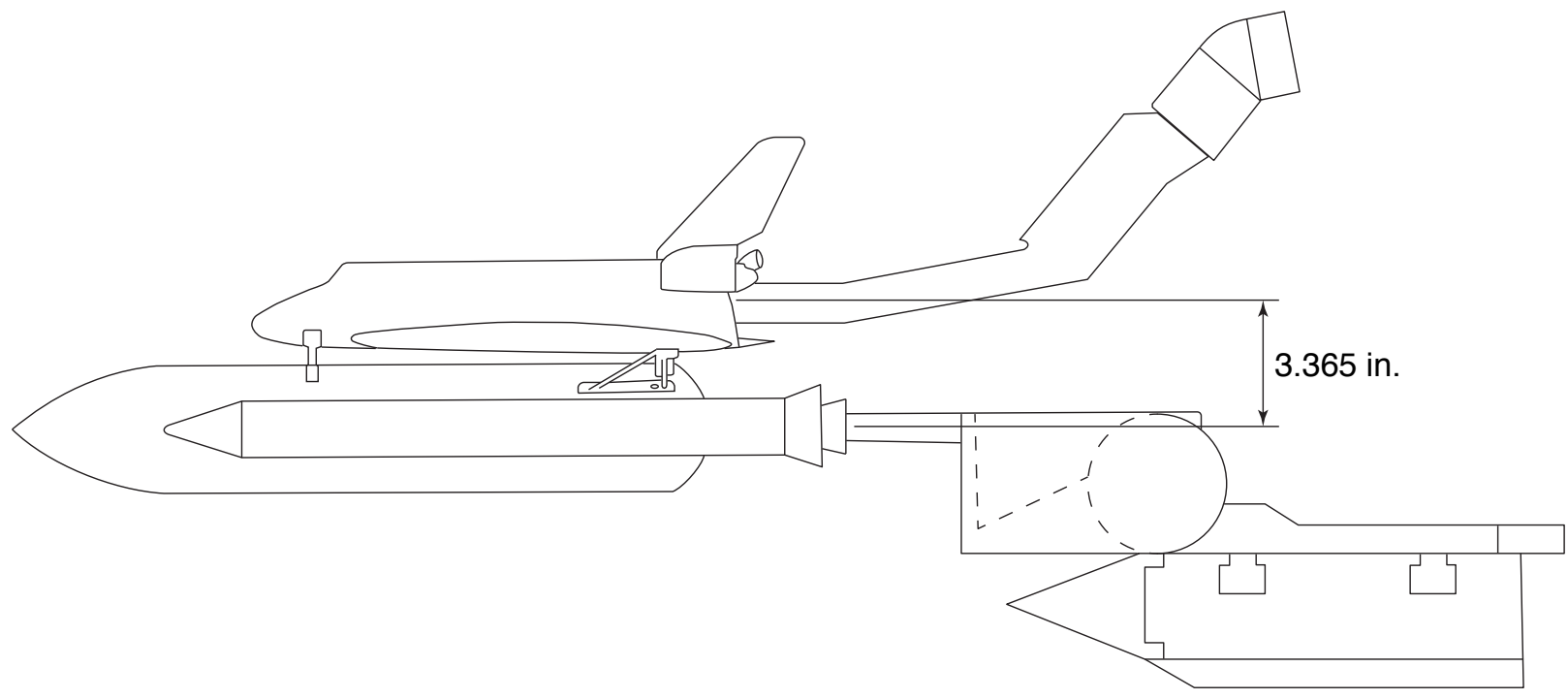

Figure 30. Sketch of Shuttle booster separation test setup. Adapted from NASA CR-151381. 